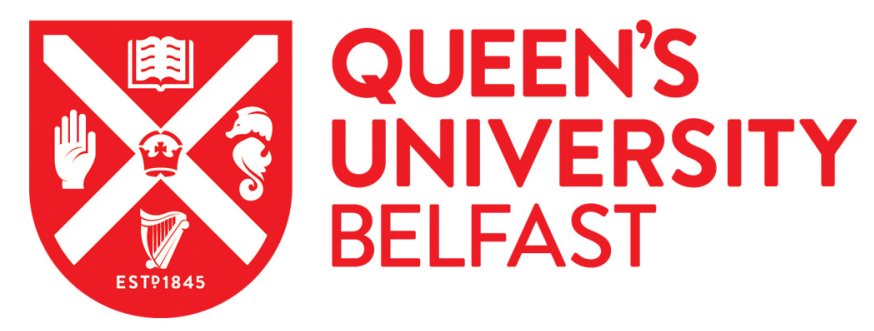

\title{
Integrated Velocity Prediction Method and Application in Vehicle- Environment Cooperative Control Based on Internet of Vehicles
}

Zhang, Y., Chen, Z., Li, G., Liu, Y., Huang, Y., Cunningham, G., \& Early, J. (2022). Integrated Velocity Prediction Method and Application in Vehicle-Environment Cooperative Control Based on Internet of Vehicles. IEEE Transactions on Vehicular Technology, 71(3), 2639-2654. https://doi.org/10.1109/TVT.2021.3139367

Published in:

IEEE Transactions on Vehicular Technology

Document Version:

Peer reviewed version

Queen's University Belfast - Research Portal:

Link to publication record in Queen's University Belfast Research Portal

Publisher rights

(c) 2021 IEEE.

This work is made available online in accordance with the publisher's policies. Please refer to any applicable terms of use of the publisher.

\section{General rights}

Copyright for the publications made accessible via the Queen's University Belfast Research Portal is retained by the author(s) and / or other copyright owners and it is a condition of accessing these publications that users recognise and abide by the legal requirements associated with these rights.

Take down policy

The Research Portal is Queen's institutional repository that provides access to Queen's research output. Every effort has been made to ensure that content in the Research Portal does not infringe any person's rights, or applicable UK laws. If you discover content in the Research Portal that you believe breaches copyright or violates any law, please contact openaccess@qub.ac.uk. 


\title{
Integrated Velocity Prediction Method and Application in Vehicle-Environment Cooperative Control Based on Internet of Vehicles
}

\author{
Yuanjian Zhang, Member, IEEE, Zheng Chen, Senior Member, IEEE, Guang Li, Senior Member, IEEE, \\ Yonggang Liu, Senior Member, IEEE, Yanjun Huang, Member, IEEE, Geoff Cunningham, and Juliana Early
}

\begin{abstract}
Rapid progress has been gained in the field of advanced communication technologies, which also promote parallel developments in the Internet of Vehicles (IoVs). In this context, vehicle-environment cooperative control can be integrated into next-generation vehicles to further improve the vehicle's performance, in particular energy efficiency. Accurate prediction of future velocity profiles on basis of IoVs can be a critical breakthrough, which can contribute much to vehicle operation efficiency promotion. In this paper, an integrated velocity prediction (IVP) method fully taking advantage of IoVs is proposed and demonstrated through a case study. In the IVP method, both the macroscopically and microscopically predicted velocity profiles are considered. The macroscopic velocity profiles are predicted via traffic flow analysis (TFA) in multi-access edge computing units (MECUs) which are situated alongside the route. Microscopic velocity profiles are forecasted through Mondrian forest (MF) algorithm in the on-board vehicle control unit (VCU). Final velocity prediction is generated through combination of the macroscopic and microscopic profiles in frequency domain in onboard VCU through fast Fourier transform (FFT) and inverse FFT. A case study validates the distinguished performance of IVP method and demonstrates its significant contribution to vehicle performance improvement. ${ }^{1}$
\end{abstract}

Index Terms-Velocity prediction, multi-access edge computing units (MECUs), traffic flow analysis (TFA), Mondrian forest (MF), Fast Fourier Transform (FFT).

\section{INTRODUCTION}

耳, MERGING development of Internet of Vehicles (IoVs) is providing a vast of opportunities for the development of novel solutions which can be implemented to improve vehicle's operation performance $[1,2]$. The superior communication ability which can be achieved through IoVs has accelerated the progress in vehicle-environment cooperative control solutions, such as multi-access edge computing (MEC) [3, 4]. By incorporating more environmental information, the vehicle performance can be promoted significantly. However, accurate vehicle velocity prediction remains one of the key bottlenecks in fully exploiting vehicle-environment control and needs to be

This work was supported in part by the National Natural Science Foundation of China (No. 61763021 and 51775063), and in part by the EU-funded Marie Skłodowska-Curie Individual Fellowship (No. 845102) (Corresponding Authors: Zheng Chen and Yonggang Liu)

Y. Zhang is with the Department of Aeronautical and Automotive Engineering, Loughborough University, Loughborough LE11 3TU, UK. (y.y.zhang@lboro.ac.uk)

Z. Chen is with the Faculty of Transportation Engineering, Kunming University of Science and Technology, Kunming, 650500, China, and with the School of Engineering and Materials Science, Queen Mary University of London, London, E1 4NS, United Kingdom. (chen@kust.edu.cn)

Copyright (c) 2015 IEEE. Personal use of this material is permitted. However, permission to use this material for any other

purposes must be obtained from the IEEE by sending a request to pubs-permissions@ieee.org.

efficiently tackled $[5,6]$. As the accuracy of velocity prediction will inevitably influence the effectiveness and viability of vehicle-environment cooperative control, there is significant effort being made to develop reliable precise forecasting methods. Yet, the prediction accuracy needs to be balanced against the computational intensity and algorithmic complexity, in order that the prediction algorithm can be implemented in real time.

To now, there has been significant progress in the field of vehicle velocity prediction. The most ordinary methods can be divided into two types: the traffic data based general prediction methods and the driving behavior based specific prediction methods. For the traffic data based method, the generated profiles typically rely on variation trends either observed in historical data that are acquired from traffic passing through a route segment, or by using historical trends in conjunction with instantly acquired traffic data combined with statistical analysis [7] or traffic flow analysis methods [8]. For example, based on the shared historical traffic data, vehicle future velocity is predicted in [8] using a traffic flow model which was then considered as a reference trajectory for facilitating optimal energy management in plug-in hybrid electric vehicles (PHEVs). The general velocity prediction methods universally assume that the vehicles drive in stable patterns and are coordinated with the surrounding vehicles on route. On this account, they are often treated as macroscopic methods. However, the differentiated performance among vehicles is seldom included in these methods, and it is intractable for general prediction methods to account for microscopic behaviors. For the driving behavior based specific velocity prediction methods, tools such as Markov chain (MC) [9] and neural network $(\mathrm{NN})$ [10] analysis are progressively adopted to provide enhanced insight into these historical datasets, allowing the exploration of microscopic behaviors. Nevertheless, the relative influence on future driving from environment, is only

G. Li is with the School of Engineering and Materials Science, Queen Mary University of London, London, E1 4NS, United Kingdom. (g.li@qmul.ac.uk).

Y. Liu is with the State Key Laboratory of Mechanical Transmissions \& School of Automotive Engineering, Chongqing University, Chongqing, 400044, China. (andylyg@umich.edu)

Y. Huang is with the School of Automotive Studies, Tongji University, Shanghai, 201804, China. (huangyanjun404@gmail.com)

G. Cunningham and J. Early are with the School of Mechanical and Aerospace Engineering, Queen's University of Belfast, BT9 5AG, Northern Aerospace Engineering, Queen's University of Belfast,
Ireland. (G.Cunningham@qub.ac.uk, j.early@qub.ac.uk). 
roughly involved. In addition, most of these methods focus on the accuracy of velocity prediction and fail to consider the computational intensity incurred in real-time application.

Velocity prediction has gradually moved from individual traffic flow analysis to a subtask of vehicle control strategy development activities. Relevant to the current work, the energy management of PHEVs can be greatly enhanced by integrating these techniques, as they allow for deeper insight into the driving conditions and therefore tuning of control parameters specific to their intended driving environments [6, 11]. Moreover, with the move towards increasing automation in the automotive domain, velocity prediction can contribute to generation of the reference trajectories during adaptive cruise [12] or automated braking processes [13]. There are also multiple applications within vehicle dynamic control - the use of velocity prediction in lateral/longitudinal traction control has been demonstrated in [14, 15].

Motivated by the literature review, vehicle performance is typically a function of environmental conditions and individual driving behaviors. The environmental conditions typically impose impact at macroscopic level (for instance, single/dual carriageway, or dry/wet weather conditions), where behavioral changes will typically be observed on all vehicles. Individual driving behaviors (how an individual vehicle will behave) typically determine the detailed maneuvers, influencing the microscopic level. Given the valuable information that is contained within both views, vehicle velocity predictions, which are based on a synthesis of macroscopic and microscopic predictions under the same scenario, shows great potential to yield improvements in accuracy.

Previous work presents an initial hybrid velocity prediction framework based on an advanced joint-prediction framework [16], where a combination of macroscopic and microscopic velocity prediction is attained through linear regression. In it, the prediction of macroscopic velocities incorporates the environmental impact; however, the vision on general traffic flow is quite scabrous for more precise prediction. The MCbased method used to generate the microscopic velocity prediction is not able to tackle the uncertainty and variability which inevitably impacts on the stochastic time-varying behaviours. The adjusted weights on the two velocities in linear regression assure the prediction accuracy compulsorily while possibly scarifying some valuable information that is connected with environment and driving behaviours. To mitigate these shortcomings, a novel integrated velocity prediction (IVP) method is advanced based on a MEC framework in IoVs, which is developed based on our former progress with better performance in real-time application. By leveraging the MECbased prediction framework, the computational intensity of onboard velocity prediction is reduced. The main contributions of this work are summarized as below:

1) An improved optimal velocity model (iOVM) is exploited with the improved macroscopic analysis on vehicle future driving, compared with the baseline statistical methods.

2) Mondrian forest (MF), as a representative of online random forests (RFs), is successfully adopted for rapid and representative regression of microscopic velocity predictions.

3) The fast Fourier transform (FFT) and inverse FFT can be used to combine macroscopic and microscopic predicted velocities into a velocity prediction results in frequency domain. The synthesis in frequency domain avoids information loss and frequent parameter tuning, facilitating real-time implementation.

4) A case study is conducted to demonstrate how a brandnew velocity prediction method that leverages the IoVs can be developed to enable the improved energy management in PHEVs.

The remainder of this paper is organized as follows. Section II provides the detailed description of the new velocity prediction method development. The case study demonstrating the application to energy management of a PHEV is discussed in Section III. Section IV details the prediction results and the positive effect of accurate velocity prediction by the proposed method in promoting energy management performance of PHEV. Finally, Section V overviews the final conclusions.

\section{Novel Velocity Prediction Method Development}

\section{A. The MEC based Framework for Velocity Prediction}

A MEC-based IoVs system is shown in Fig. 1, where a multiaccess edge computing unit (MECU) is mounted at the base station (BS) and owns strong computational capability. In this framework, partial control tasks can be offloaded to MECUs [17]. By positioning these MECUs on road segments, traffic status data such as global position system (GPS) coordinates, vehicle speeds, distances to forward vehicles and travel destination information shared by the volunteered vehicles on route segments can be collected and processed by MECUs. Relevant information from MECUs can then be disseminated to the vehicle, thus reducing the computational burden on the vehicle on-board hardware [18].

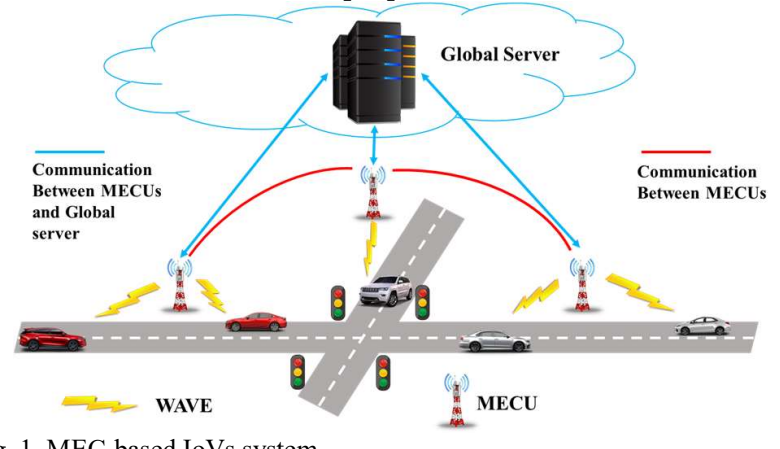

Fig. 1. MEC-based IoVs system.

In the MEC based framework, communication is assumed to be attained through millmetre wave (mmWave, better known as $5 \mathrm{G})$ communication due to its ultra-wide band [19]. The BSs, connecting with multiple antennas, are equipped with a single ratio frequency $(\mathrm{RF})$ chain to reduce hardware complexity and energy consumption [19]. In each MECU, the total computing capacity is modelled as 20 Giga cycle/s [20].

\section{B. The Novel Velocity Prediction Method}

The novel velocity prediction method proposed is based on the MEC framework described in Section II. Velocity prediction is achieved through cooperation between MECUs 
and the on-board vehicle control unit (VCU) in real time, which provides a pathway for enhanced computational efficiency beyond that which would be achievable through only the onboard vehicle hardware in isolation. An overview of the proposed IVP method is shown in Fig. 2. The predicted velocity profile is synthesized from the macroscopic and microscopic velocity forecast. The designed synthesis is inspired from the noticeable advantage and disadvantage of the macroscopic and microscopic forecast. The macroscopic velocity prediction can vision picture of the general traffic flow trend but cannot inspect the details of single vehicle. The microscopic velocity prediction can inform detailed behaviours of individual vehicle but lacks full visibility of dynamics of vehicle swarm. The MECU provides the macroscopic velocity profile based on historical data at that road segment location. A microscopic velocity profile is simultaneously generated by the on-board VCU. These two profiles are combined in the frequency domain and finally transformed using the inverse FFT into time domain to generate the final 'mesoscopic' velocity prediction for vehicle control.

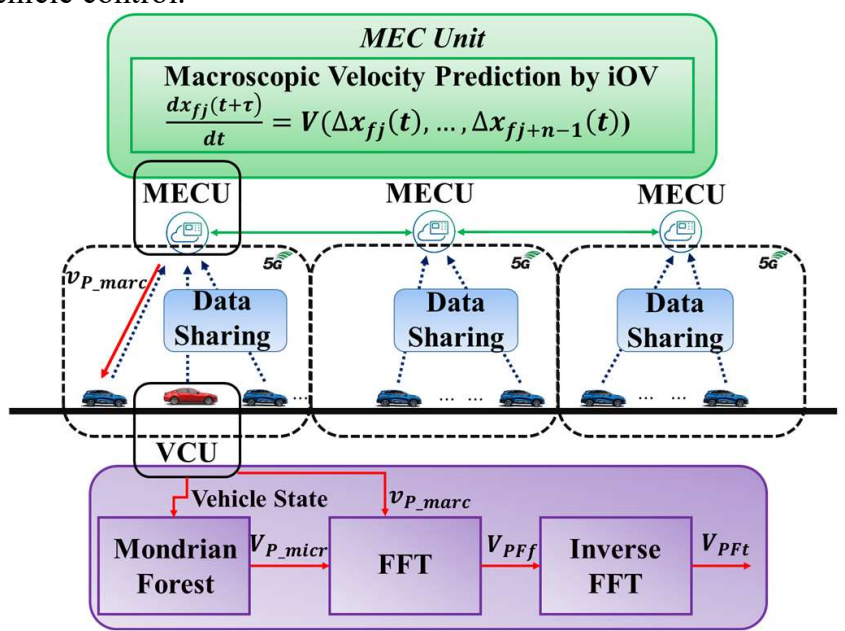

Fig. 2. Illustration of the IVP process.

In the current work, the macroscopic velocity is predicted through the improved OV model held in MECUs with refined macro-description ability. The microscopic velocity prediction is calculated via MF in the on-board vehicle VCU with the evolved capacity in addressing the uncertainty and variability in individual future driving. The two velocity predictions are combined into the final predicted velocity profile in frequency domain via FFT and inverse FFT in the on-board VCU. The synthesis in frequency domain avoids information compression and promotes retention of the original information related to both environmental and driving behaviours. Through the distributed velocity prediction, the computational burden originally imposed on on-board VCU is partially mitigated by MECUs, accelerating the prediction process in real time. It is worth noting that the velocity prediction method proposed in this paper is only for vehicle longitude driving in random swarm with stable driving status, and vehicle lateral dynamic is not taken in consideration. The primary role of the forecasted velocity lies in enlightening energy management strategy to make optimal decisions in energy distribution within powertrain. With more control inputs and constraints on vehicle dynamic and platoon stability, the proposed methods can predict velocity profiles for autonomous vehicle in both individual and platoon level, and this will be studied carefully in our future investigation. Even though the macroscopic velocity prediction in this paper has considered safety issues during car following scenarios, it only includes soft constraints, as the studied scenarios are constrained to stable vehicle driving by following forward vehicles in randomly distributed vehicle swarm without adversarial behaviors from background, rather than in platoon. The detailed methods to predict and combine velocities will be described in the following section.

\section{a. The Macroscopic Velocity Prediction by iOVM}

On-road surrounding vehicles and road conditions can greatly affect the target vehicle driving behaviour. While the behaviour of the vehicle driver will typically only impact on the local microscopic vehicle behaviour, environmental conditions will have a macroscopic impact on vehicle future dynamics. For a target vehicle driving in a constant traffic flow, it can be assumed that it will frequently adjust its speed to maintain safe speeds and separation distances. This need for adjustments at the microscopic level can be due to changes in the surrounding environment (for instance, road conditions), and as a result there is a strong coupling between the microscopic and macroscopic behaviour. For this reason, an effective model should be capable of efficiently incorporating environmental information with induced driving behaviours. The optimal velocity model [21], as one of the promising traffic flow models, shows the remarkable potential in processing the incorporation. The general expression of the optimal velocity model can be formulated as:

$$
\left\{\begin{array}{l}
\ddot{x}_{n}=a\left[V\left(\Delta x_{n}(t)\right)-\dot{x}_{n}(t)\right] \\
\Delta x_{n}(t)=x_{n+1}(t)-x_{n}(t)
\end{array}\right.
$$

where $n$ is the vehicle number, $x_{n}$ is the coordinate of the $n$th vehicle, $a$ is the parameter related to driver sensitivity, and $V$ is the legal velocity. The legal velocity is the reference for drivers to control vehicle acceleration and braking in order to maintain a safe distance from preceding vehicle without causing instability in traffic flow. It is a function of the derivation on relative distance to forward vehicles. During driving on route segment with random distribute vehicle swarm, drivers tend to keep forward traffic flow without causing adversarial interconnection with background vehicles; thus, a reference velocity is suggested to follow. Legal velocity acts as a critical index to assist drivers driving stably in randomly distributed vehicle swarm, and can reveal macroscopic performance of traffic flow to a great extent. As such, legal velocity is adopted to describe macroscopic traffic flow moving feature.

In IoVs, vehicle status on a route segment is assumed to be shared via vehicle to vehicle $(\mathrm{V} 2 \mathrm{~V})$ and vehicle to infrastructure (V2I) communication [22]. The distance to forward vehicles and relative speeds between vehicles on a certain route segment can be measured by radars on vehicles. To improve rationality and accuracy on macroscopic velocity, the relative speed to forward vehicles is also incorporated in the calculation of legal velocity. On the basis, the following relationships can be formulated, as: 


$$
\frac{d x_{j}(t+\tau)}{d t}=V\left(\sum_{l=0}^{n} \Psi_{l} \Delta x_{j+l}(t), \sum_{l=0}^{n} \Gamma_{l} \Delta v_{j+l}(t)\right)
$$

where

$$
\begin{gathered}
\left\{\begin{array}{l}
\Delta x_{j+l}(t)=x_{j+l+1}(t)-x_{j+l}(t) \\
\Delta v_{j+l}(t)=v_{j+l+1}(t)-v_{j+l}(t)
\end{array}\right. \\
\Psi_{l}=\left(\frac{1}{6}\right)^{l+1}, \Gamma_{l}=\left\{\begin{array}{l}
\frac{5}{6^{l}}, h \neq n \\
\frac{1}{6^{l-1}}, h=n
\end{array}\right.
\end{gathered}
$$

In (2), $\Delta x_{j}$ denotes the headway of $j$ th vehicle to the forward vehicle, $\Delta v_{j}$ is the relative speed of $j$ th vehicle to the forward vehicle, $l$ is the number of vehicles that are considered, $\Psi_{l}$ and $\Gamma_{l}$ are ratios weighing the impact of forward vehicles, and $\tau$ is the time lag before driver adjusts the speed to reach the legal velocity $V$. The relationship presented in (2) reveals how drivers adjust the corresponding behaviors to keep stable driving in the randomly distributed vehicle swam according to relative speed and distance to forward vehicles. Regardless of the value of $\tau$, equation (2) can be rewritten using the Taylor expansion, as:

$$
\frac{d v_{j}(t)}{d t}=a\left[V\left(\sum_{l=0}^{n} \Psi_{l} \Delta x_{j+l}(t)\right)-\frac{d x_{j}(t)}{d t}\right]+k \sum_{l=0}^{n} \Gamma_{l} \Delta v_{j+l}(t)
$$

where $k$ denotes the sensitivity to the relative velocities of the forward vehicles, and relates to how driver reacts to inconformity with the whole vehicle swam. To reach consistent status with following vehicles in randomly distributed swam for safe driving, drivers manipulate vehicles with different accelerations and decelerations, which are described by $a$ and $k$. According to (2) and (5), the legal velocity of each vehicle can be written as:

$$
\begin{aligned}
V\left(\sum_{l=0}^{n} \Psi_{l} \Delta x_{j+l}(t)\right)= & \tanh \left(\sum_{l=0}^{n} \Psi_{l} \Delta x_{j+l}(t)-d_{h s}\left(\Delta v_{j+l}(t)\right)\right) \\
& +\tanh \left(d_{h s}\left(\Delta v_{j+l}(t)\right)\right.
\end{aligned}
$$

where $d_{h s}$ is the safe headway under different relative speeds. In the calculation of legal velocity, the noise, disturbance and data communication may result in errors in the estimated distance to forward vehicles, thus discounting the calculation of legal velocity. Hence, the collected data shared by vehicles in IoVs will be processed by Kalman filter [23] before implementing in legal velocity estimation to reduce the interference on final output and improve the observing accuracy. To attain the legal velocity based on (6), the distance headway and relative speed of target vehicle to forward vehicles can be calculated according to the measure of sensors installed in each vehicle. The safe headway $d_{h s}$ in (6) can be calculated,

$$
d_{h s}\left(\Delta v_{j+l}\right)=S_{\text {stop }}+k_{v} \times \Delta v_{j+l}
$$

where $S_{\text {stop }}$ is the desired distance to forward vehicle when target vehicle stops, $k_{v}$ is a proportional constant. Besides, $\Psi_{l}$ is carefully tuned to make sure that the obtained legal velocity is rational by the tanh function presented in (6). The number of forward vehicles that are included in calculation is also limited to 5 to exclude additional disturbance. Based on the given headway distance, relative speeds and safe headway, the legal velocity can be determined. Each vehicle on a certain route segment can estimate the legal velocity through on-board hardware and share the estimation to the closest MECU through V2I communication. The MECU that covers the specific route segment calculates the macroscopic velocity of the specified route segment, as:

$$
V_{p_{-} \text {macro_ } r}=\frac{1}{N} \sum_{i=1}^{N} V_{i}
$$

where $N$ is the number of vehicles on route segment, and $V_{i}$ is the legal velocity of each vehicle obtained by (6). The travel time $t_{r}$ on one route segment can be expressed as:

$$
t_{r}=\frac{d_{r}}{V_{p_{-} \text {macro_r }}}
$$

where $d_{r}$ is the length of a known route segment that is shared to the MECUs previously. The macroscopic velocity profile of each route segment is shared among MECUs. The target vehicle requests the predicted velocity profile within the predefined length from the closest MECU, together with the information about the proposed route. The MECU collects the macroscopic velocity profiles for different route segments based on the provided information, and the macroscopic velocity profile $V_{p_{-} \text {macro }}$, will be generated according to the given prediction horizon and calculated travel times in the specific route segment. The legal velocity and calculated macroscopic velocities of each route segment are updated every $1 \mathrm{~s}$. The communication time in V2V and V2I communication under the prescribed tasks are obviously much less than $1 \mathrm{~s}$ within the service range of each MECU or between two MECUs due to the strong communication and computation capabilities in the advanced IoVs, which can be neglected in the prediction process and further evaluation [24]. In this paper, the service radius of each MECU is $500 \mathrm{~m}$. The length of the route segments will affect the predicted velocity over the chain of segments. For each segment, a predicted velocity is provided, which will result in a step sequence when all the segments are considered together (assuming that the velocity prediction will change in each segment). This is not consistent with the expected continuous velocity variation. To solve the problem, the Chebyshev lowpass filter [25] is employed, and the smoothed macroscopic velocity profile is sent back to the target vehicle via V2I communication for further processing.

\section{b. The Microscopic Velocity Prediction by Online RF}

The RF is the modified version of the Bagging method, which has been widely accepted as an ensemble learning method [26]. In the RF based regression analysis, the decisiontree based training process is implemented, and the prediction result is obtained by averaging the output of each decision-tree. To achieve better performance in on-board VCUs in real time, the Mondrian forest (MF) [27], as one of evolved online RFs, has been selected in this study. The construction of MF is similar to the RF. For a regression problem, given the training data $\mathrm{D}_{1: N}:=\left(X_{1: N}, Y_{1: N}\right)$, a collection of Mondrian trees 
$\left(T_{1}, T_{2}, \ldots T_{M}\right)$ can be randomly constructed [28]. The prediction by each Mondrian tree is the distribution $p_{T_{m}}\left(y \mid x, \mathrm{D}_{1: N}\right)$ over output label $y$ for the test input $x$. The prediction by MF is the average of each Mondrian tree that can be expressed as:

$$
y_{T_{m}}\left(x, \mathrm{D}_{1: N}\right)=\frac{1}{M} \sum_{m=1}^{M} p_{T_{m}}\left(y \mid x, \mathrm{D}_{1: N}\right)
$$

When $M$ approaches infinity, the average of each Mondrian tree converges to:

$$
y_{\infty}\left(x, \mathrm{D}_{1: N}\right)=\mathrm{E}_{T \sim \mathrm{MT}\left(\lambda, \mathrm{D}_{1: N}\right)}\left[p_{T}\left(y \mid x, \mathrm{D}_{1: N}\right)\right]
$$

where $\operatorname{MT}\left(\lambda, \mathrm{D}_{1: N}\right)$ can be expressed as:

$$
\operatorname{MT}\left(\lambda, \mathrm{D}_{1: N}\right)=p_{T_{m}}\left(y \mid x, \mathrm{D}_{1: N}\right)
$$

In the online learning process, the training samples are presented in a sequence of trials. At the $N+1$ th iteration, each Mondrian tree $T$ will be updated to integrate the next sample by randomly choosing an extended tree from the distribution $T^{\prime}$. Based on the features of the Mondrian process, the distribution of $T^{\prime}$ can be expressed as:

$$
\left\{\begin{array}{l}
T \sim M T\left(\lambda, \mathrm{D}_{1: N}\right) \\
T^{\prime} \sim \operatorname{MTx}\left(\lambda, T, \mathrm{D}_{1: N}\right)
\end{array} \rightarrow T^{\prime} \sim \operatorname{MT}\left(\lambda, \mathrm{D}_{1: N}\right)\right.
$$

According to (13), the distribution of the Mondrian tree is independent of the data observing order. In fact, the distribution of a Mondrian Tree trained with the incremental form is the same with that trained in a batch form under the given training data [29]. In the MF, the Mondrian tree is a rooted-binary tree $\mathrm{T}$ in which there are leaf, internal and root nodes [27]. The root and leaf nodes can be denoted by $\mathrm{E}$ and $\mathrm{L}(\mathrm{T})$, respectively. For each internal node $j \in \mathrm{T} \backslash \mathrm{L}(\mathrm{T})$, the child nodes of the given internal node can be expressed by $\mathrm{G}(j)$ and $\mathrm{C}_{r}(j)$. Each internal node is associated with a portion of input data $B_{j} \in \mathrm{R}^{D}$, where $B_{\mathrm{E}}:=\mathrm{R}^{D}$. In the training process, each internal node $j$ is assigned a split action $\mathrm{S}\left(\delta_{j}, \xi_{j}\right)$, $\delta_{j}=1,2, \ldots, D . \xi_{j}$ reveals the split location among the dimension $\delta_{j}$. The spilt can be further defined as:

$$
\left\{\begin{array}{l}
B_{\mathrm{C}_{(}(j)}:=\left\{x \in B_{j}: x_{\delta_{j}} \leq \xi_{j}\right\} \\
B_{\mathrm{C}_{r}(j)}:=\left\{x \in B_{j}: x_{\delta_{j}}>\xi_{j}\right\}
\end{array}\right.
$$

In the reference velocity estimation, the input of MF is the history velocity profile $V_{h_{-} i}\left(v_{h_{-} i}, v_{h_{-} i-1}, \ldots, v_{h_{-} i-n}\right)$, where $n=1,2, \ldots, k$ denotes the time step in the history data, $i$ is the current time step; and the output of MF is the velocity difference of the neighboring time step $\Delta V_{p_{-} i}$ $\left(\Delta v_{p_{-} i+1}, \Delta v_{p_{-} i+2}, \ldots, \Delta v_{p_{-} i+m}\right)$, where $m=1,2, \ldots, l$ is the time step of future driving. The estimated microscopic velocity $V_{p_{-} m i c r o \_} i+m$ at time $i+m$ can be expressed as:

$$
V_{p_{-} \text {micro } \_i+m}=V_{i}+\Delta v_{p_{-} i+1}+\ldots+\Delta v_{p_{-} i+m}
$$

As shown in (15), the future microscopic velocity is based on the difference between the neighboring time steps, rather than actual values. This is due to the average estimation in MF - it is unable to extrapolate. Specifying velocity difference rather than actual velocities as the output of MF can improve the adaptability of MF to uncertainties in regression analysis. For the construction of the microscopic velocity profile, $15 \mathrm{~s}$ of the detailed historical velocity and a prediction horizon of $10 \mathrm{~s}$ are assumed. The time step is set to $1 \mathrm{~s}$ in this study. During model training when predicting the microscopic velocity, the data comes from vehicle tests in practical situation. However, there also exists noticeable data noise caused by improper measurement from speed sensors and signal mutual interference during data collection via central aera network (CAN) channel. To ensure training effectiveness, Kalman filter [23] is applied to refine the collected training data.

\section{c. The Synthesis into Finally Predicted Velocity}

After obtaining the predicted macroscopic and microscopic velocity profile, the final velocity profile is synthesized in the on-board VCU through FFT and inverse FFT. The general process of velocity synthesis is shown in Fig. 3. The information from the two different velocity predictions is originally expressed in time domain. Using FFT, the timedomain velocity profiles will be converted into the frequency domain, resolving it into waves with different frequencies. The wave belonging to the macroscopic velocity profile is combined with the corresponding wave attached to the microscopic velocity profile at the same frequency. After combing the two waves, the final predicted velocity profile is transformed back to the time domain through the inverse FFT. The equation to describe the synthesis can be formulated as:

$$
\begin{aligned}
& V_{P F}=i n v F F T\left[\mathbf{V}_{P F_{-} f d}\right] \\
& =i n v F F T\left[F F T\left[\mathbf{V}_{p_{-} \text {macro }}\right]+F F T\left[\mathbf{V}_{p_{-} \text {micro }}\right]\right]
\end{aligned}
$$

where $\mathbf{V}_{P F_{-} f d}$ is the synthetic velocity in frequency domain. The FFT and inverse FFT are evolved from the discrete-time Fourier transform (DFT) and inverse DFT [30]. Compared with the DFT and inverse DFT, FFT and inverse FFT possess less computational complexity and accelerated computation abilities [31]. The equations in terms of DFT and inverse DFT can be written as:

$$
\left\{\begin{array}{l}
y(k)=\sum_{m=0}^{N-1} x(m) e^{\frac{-2 \pi i k m}{N}}, 0 \leq k \leq N-1 \\
x(m)=\sum_{m=0}^{N-1} y(k) e^{\frac{2 \pi i k m}{N}}, 0 \leq m \leq N-1
\end{array}\right.
$$

where $N$ is the number of signal points. According to (17), the computation complexity is $\mathrm{O}\left(N^{2}\right)$. In FFT, assuming $N=2^{r}$, DFT can be rewritten as: 


$$
\begin{aligned}
& y(k)=\sum_{m=0}^{N / 2^{-1}} x(2 m) e^{-\frac{2 \pi i k(2 m)}{N}}+\sum_{m=0}^{N / 2^{-1}} x(2 m+1) e^{-\frac{2 \pi i k(2 m+1)}{N}} \\
& =\underbrace{\sum_{m=0}^{N / 2} x(2 m) e^{-\frac{2 \pi i k m}{N / 2}}}_{A_{k}}+e^{-\frac{2 \pi i k}{N}} \underbrace{\sum_{m=0}^{N / 2^{-1}} x(2 m+1) e^{-\frac{2 \pi i k m}{N / 2}}}_{B_{k}}
\end{aligned}
$$

where $A_{k}$ denotes the even-numbered data points, $B_{k}$ is the odd-numbered data points. Equation (18) can be rewritten as:

$$
y(k)=A_{k}+e^{-\frac{2 \pi i k}{N}} B_{k}
$$

Replacing $k$ in (18) with $k+\frac{N}{2}$, we can get:

$$
\left\{\begin{array}{l}
e^{-2 \pi i(k+N / 2)(2 m) / N}=e^{-2 \pi i k^{(2 m)} / N} e^{-2 \pi i m}=e^{-2 \pi i k^{(2 m)} / N} \\
e^{-2 \pi i(k+N / 2) / N}=e^{-2 \pi i k / N} e^{-\pi i k}=-e^{-2 \pi i k / N}
\end{array}\right.
$$

According to (20), equation (18) can be simplified as:

$$
\left\{\begin{array}{l}
y(k)=A_{k}+e^{-\frac{2 \pi i k}{N}} B_{k} \\
y\left(k+\frac{N}{2}\right)=A_{k}-e^{-\frac{2 \pi i k}{N}} B_{k}
\end{array}\right.
$$

The even and odd-numbered terms in DFT can be calculated by (21). The result of DFT is acquired by combining the calculated even and odd-numbered terms. Assume that $S_{N}$ is the number of steps in DFT described in (18), as:

$$
S_{N}=\left\{\begin{array}{cc}
0 & N=0 \\
2 S_{\frac{N}{2}}+\frac{N}{2} & N>1
\end{array}\right.
$$

By computing the sequence in (21), the number step $S_{N}^{\prime}$ of the process in (21) can be written as:

$$
S_{N}^{\prime}=\frac{N}{2} \log _{2} N
$$

Accordingly, the computational complexity of the improved DFT is $\mathrm{O}(n \log n)$, lower than that by the original DFT. The improved DFT is also called FFT. Also, the inverse FFT can be calculated by the similar process with that used for FFT. During the velocity synthesis, the prediction length of the macroscopic velocity profile is the same with that of the microscopic profile. In the velocity prediction, the data are discretized by an equalwidth interval method [32]. The data for macroscopic and microscopic predicted velocity are discretized with the same time step (1s). Indeed, the discretization can affect velocity prediction accuracy significantly. With smaller discretization step, data sampling frequency in macroscopic velocity prediction cannot satisfy the precision requirement, leading to poor macroscopic velocity prediction. In addition, smaller discretization step will increase energy consumption in communication process in IoVs [33], and will be also not favored in practical application. With larger discretization step, some behaviors information in future driving might not be captured, worsening accuracy prediction either.

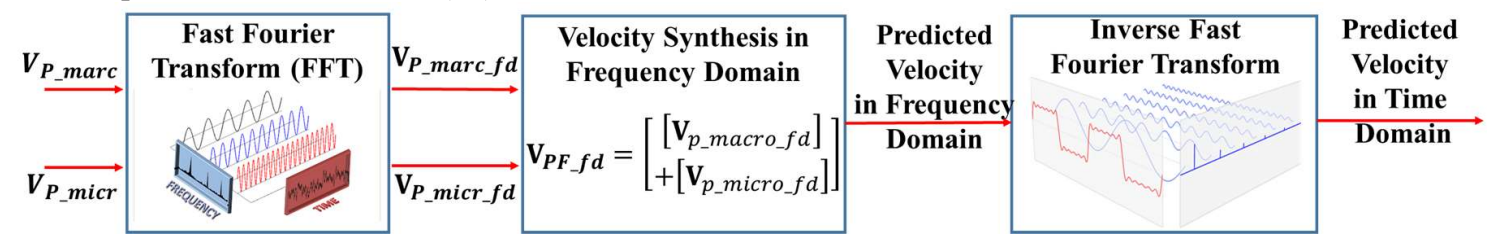

Fig. 3. Illustration on velocity synthesis process.

\section{CASE STUdy: InVESTIGATION ROLE OF IVP IN IMPROVING ENERGY MANAGEMENT PERFORMANCE OF PHEV}

As is discussed in Section I, effective energy management strategies for PHEVs are highly dependent on future driving conditions and provide a preferable chance to evaluate the developed IVP method. A case study based on the MPC strategy that incorporates the IVP method for PHEV is performed. In the MPC based energy management strategy, the future velocity profile obtained by the IVP method is regarded as the reference for the speed tracking based control in the receding horizon optimization of MPC.

\section{A. PHEV Configuration}

The powertrain configuration of the studied PHEV is illustrated in Fig. 4. The hybrid powertrain consists of internal combustion engine (ICE), generator and motor. Through the cooperative work among ICE, generator, and motor, the PHEV can operate in several modes including all-electric mode (also known as EV mode), serial mode and parallel mode. In allelectric mode, the battery pack provides all the propelling energy. In serial mode, the auxiliary power unit (APU), composed of ICE, generator and battery pack supply the required tractive energy. In parallel mode, the ICE, together with the motor, drives the vehicle directly. The mode transition is attained by governing the engagement/ disengagement of clutch installed between ICE and motor.

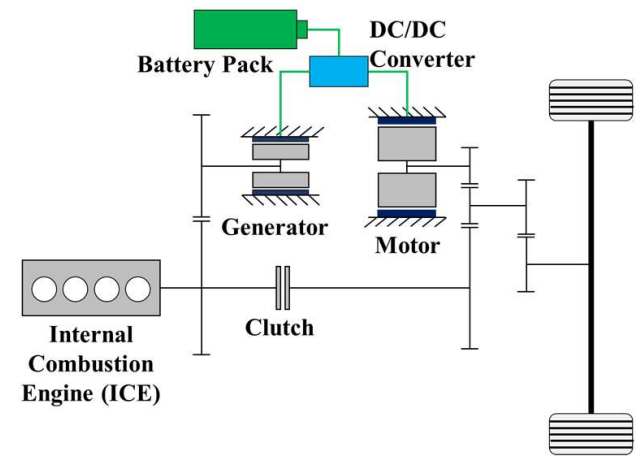

Fig. 4. The schematic of the studied PHEV configuration.

A forward model is built in Matlab/Simulink to support the development of MPC based energy management strategy, and there exist four sub-modules in the built model: driver, plant, controller, and CAN bus. The driver module interprets driving behavior and outputs the specific driving requirement 
(power/torque demand) to the controller module. The controller module determines the most suitable operation mode based on driving demand and current vehicle state, and distributes energy demand among ICE, generator, and motors accordingly. The plant module dynamically responds to the given control orders transmitted from the controller module, and supplies the actual execution details.

\section{B. MPC Based Energy Management in PHEV}

The energy management in PHEV aims to distribute the energy demand among the available power sources. For efficient energy management in the studied PHEV, the target is to meet the required performance with minimum energy consumption. In the MPC based energy management strategy, the power distribution ratio $\mu_{1}$ between ICE and motor and the power distribution ratio $\mu_{2}$ between APU and motor are set to the control imputs. The vehicle speed $v$ and battery SOC are considered as the state variables. Notably, $\mu_{1}$ and $\mu_{2}$ are respectively applied in parallel and serial mode. The cost function can be expressed as:

$$
J=\int_{t_{k}}^{t_{f}} \varphi_{1} \dot{m}_{f}(t)+\varphi_{2}\left(v(t)-V_{P F}(t)\right)^{2}+\varphi_{3} \frac{P_{b a t t}(t)}{Q_{l h v}}
$$

where $\varphi_{1}, \varphi_{2}$ and $\varphi_{3}$ are the weight ratio, $\dot{m}_{f}$ is the fuel consumption rate, and $P_{\text {batt }}$ is the battery power. The fuel consumption rate can be attained by interpolating the engine fuel consumption map according to the instant engine torque and speed. The engine speed can be measured directly via the installed sensor in vehicle. Given the control input $T_{r e q_{-} d}, \mu_{1}$ and $\mu_{2}$, ICE torque $T_{\text {eng }}$ and $P_{\text {batt }}$ can be calculated as:

$$
\begin{gathered}
T_{\text {eng }}= \begin{cases}\frac{\left(1-\mu_{2}\right) T_{\text {trac }} n_{\text {wheel }}}{\eta_{t_{-} f d} \eta_{t_{-} g} \eta_{e_{-} g m} n_{\text {eng }}} & \text { Serial Mode } \\
\frac{\left(1-\mu_{1}\right) T_{\text {trac }} n_{\text {wheel }}}{\eta_{t_{-} f d} \eta_{t_{-} g 1} n_{\text {eng }}} & \text { Parallel Mode }\end{cases} \\
P_{\text {batt }}(t)= \begin{cases}\frac{\mu_{2} T_{\text {trac }} n_{\text {wheel }}}{9550 \eta_{t_{-} f d} \eta_{t_{-} g} \eta_{e_{-} b m}} & \text { Serial Mode } \\
\frac{\mu_{1} T_{\text {trac }} n_{\text {wheel }}}{9550 \eta_{t_{-} f d} \eta_{t_{-} g} \eta_{e_{-} b m}} & \text { Parallel Mode }\end{cases}
\end{gathered}
$$

where $\eta_{e_{-} b m}$ is the electric transmission efficiency between motor and battery, $\eta_{e_{-g m}}$ is the electrical transmission efficiency between generator and motor, and $\eta_{t_{-} g 3}$ is the mechanical transmission efficiency of the gear between ICE and generator. The inequality constraints assigned to the MPC based control problem can be written as:

$$
\left\{\begin{array}{l}
S O C_{\text {min }} \leq S O C(t) \leq S O C_{\max } \\
P_{\text {batt_min }} \leq P_{\text {batt }}(t) \leq P_{\text {batt_max }} \\
T_{\text {eng_min }} \leq T_{\text {eng }}(t) \leq T_{\text {eng_max }} \\
T_{\text {mot_min }} \leq T_{\text {mot }}(t) \leq T_{\text {mot_max }} \\
T_{\text {gen_min }} \leq T_{\text {gen }}(t) \leq T_{\text {gen_max }} \\
\omega_{\text {eng_min }} \leq \omega_{\text {eng }}(t) \leq \omega_{\text {eng_max }} \\
\omega_{\text {mot_min }} \leq \omega_{\text {mot }}(t) \leq \omega_{\text {mot_max }} \\
\omega_{\text {gen_min }} \leq \omega_{\text {gen }}(t) \leq \omega_{\text {gen_max }}
\end{array}\right.
$$

where the superscripts min and max denote the minimum and maximum value of each variable. $T_{m o t}$ and $T_{g e n}$ represent the torque of motor and generator, $\omega_{m o t}$ and $\omega_{\text {gen }}$ are their rotational speed, respectively. The equality constraints in the MPC have been selected to maintain the vehicle dynamics and electricity energy consumption. The chosen equality constraints can be expressed as:

$$
\left\{\begin{array}{l}
\dot{v}(t)=\frac{1}{m}\left(\begin{array}{l}
T_{\text {trac }}(t)-\frac{1}{2} C_{D} A v^{2}(t) \\
-m g f \cos \alpha(t)-m g \sin \alpha(t)
\end{array}\right) \\
S \dot{O} C(t)=-\frac{V_{o c}(t)-\sqrt{V_{o c}(t)-4 R_{\mathrm{int}}(t) P_{b a t t}(t)}}{2 R_{\mathrm{int}}(t) Q_{\text {batt }}}
\end{array}\right.
$$

After designing the control inputs, state variables, cost function, inequality and equality constraints, the MPC based control problem can be solved in the receding horizon. The general process of MPC implementation is shown in Fig. 5. The algorithm searches the optimal control inputs to obtain the minimum cost in the receding horizon, in which the state variables are updated based on the equality constraints. The predicted velocity profile based on the proposed algorithm, as the reference for tracking, directs the searching in the receding horizon. In this case, the sequential quadratic programming (SQP) [34] is considered as the solution manner.

Despite the case study in this paper that showcases the performance of the IVP method in the scope of MPC based energy management in PHEV, there is quite huge potential to apply the IVP method in other scenarios. With the IVP method, the energy management in process of adaptive cruise can be improved for the endowed more valuable information about behaviors of surrounding vehicles. The proposed IVP, after integrating velocity planning for lateral motion, can be applied in trajectory optimization for autonomous vehicles. Besides, the forecasted velocity profiles by the IVP method can be implemented in research on optimal navigation with various optimization target for autonomous electric vehicles. 


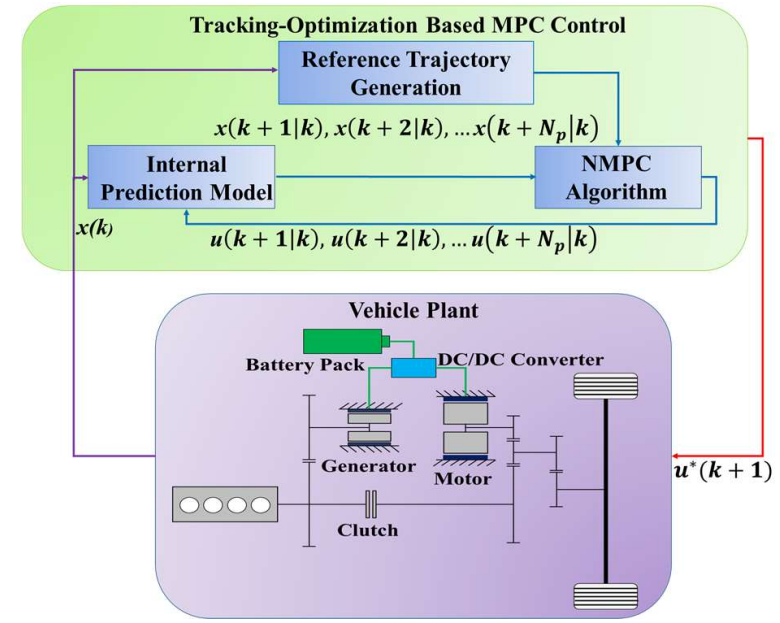

Fig. 5. The illustration on MPC implementation process.

\section{Simulation Evaluation}

In this section, the performance of the IVP method and the case study results are evaluated. To better assess the performance of the IVP method, the single iOVM, the single $\mathrm{MF}$, and the hybrid velocity profile prediction (HVPP) methods [16], back propagation neural network (BP-NN) [35], support vector machine (SVM) [36], and long short term memory network are respectively applied for comparison [37]. The data adopted for training the selected models and evaluation is derived by the method in [38] based on real traffic data that are collected on real routes. The method to derive training samples in [38] considers the driving behavior factors in vehicle driving. In the application of the IVP method, the iOVM based macroscopic velocity prediction model only predicts the legal velocity based on the shared traffic data from vehicles on route segment, while the pre-trained MF based microscopic velocity prediction obtains future driving velocities based on the input velocity profile. In the case study, the MPC based strategies with IVP and HVPP are simulated. In addition, the dynamic programming (DP) based control strategy and simple rule based control strategy, i.e., charge depletion-charge sustaining (CD$\mathrm{CS})$ scheme are also considered as comparison. In the CD-CS strategy, battery pack is the only power source when battery SOC is larger than 0.28. After battery SOC drops less than 0.28 , the vehicle operates in the CS stage, and the traction comes from both ICE and battery pack. In the evaluation, 'New CS' denotes the MPC based strategy that incorporates the IVP method, and 'Original CS' denotes the rule-based method obtained from the benchmark test. The simulation is conducted on a workstation with an Intel Xeon E3-1270@3.4 GHz and 32 Gigabytes memory. The main parameters for the studied PHEV are listed in Table I.

TABLE I

COMPONENT PARAMETERS IN THE STUDIED IPHEV

\begin{tabular}{lll}
\hline \multirow{3}{*}{ Engine } & \multicolumn{2}{l}{ COMPONENT PARAMETERS IN THE STUDIED IPHEV } \\
\cline { 2 - 3 } & Displacement & $\mathbf{2 . 0}[\mathrm{L}] \mathbf{1 6 V}$ DOHC \\
\cline { 2 - 3 } & Maximum Power & $105[\mathrm{~W}] @ 6200[\mathrm{rpm}]$ \\
\cline { 2 - 3 } Motor and & Maximum Torque & $165[\mathrm{Nm}] @ 2500 \sim 6500[\mathrm{rpm}]$ \\
\cline { 2 - 3 } Generator & Maximum Torque & $124[\mathrm{~kW}]$ \\
\hline \multirow{3}{*}{ Battery } & Type & $307[\mathrm{Nm}]$ \\
\cline { 2 - 3 } & Capacity in Ah & Lithium-ion \\
\cline { 2 - 3 } & Capacity in $\mathrm{kWh}$ & $39[\mathrm{Ah}]$ \\
\hline
\end{tabular}

\begin{tabular}{lll}
\hline & Nominal Voltage & $300[\mathrm{~V}]$ \\
\cline { 2 - 3 } & Battery Pack Resistance & $0.12[\Omega]$ \\
\cline { 2 - 3 } & Configuration & 100 cells connected in serial \\
\hline \multirow{5}{*}{ Gears } & $\begin{array}{l}\text { Gear 1 (Between ICE and final } \\
\text { drive) }\end{array}$ & $i_{\mathrm{g} 1=0.803}$ \\
\cline { 2 - 3 } & $\begin{array}{l}\text { Gear 2 (Between motor and } \\
\text { final drive) }\end{array}$ & $i_{\mathrm{g} 2=2.45}$ \\
\cline { 2 - 3 } & $\begin{array}{l}\text { Gear 3 (Between ICE and } \\
\text { generator) }\end{array}$ & $i_{\mathrm{g} 3}=1.934$ \\
\cline { 2 - 3 } & Final Drive Gear & $i_{\mathrm{g} 4}=9.665$ \\
\cline { 2 - 3 } & Efficiency of Gear 1 & 0.92 \\
\cline { 2 - 3 } & Efficiency of Gear 2 & 0.92 \\
\cline { 2 - 3 } & Efficiency of Gear 3 & 0.93 \\
\cline { 2 - 3 } & Efficiency of Final Drive Gear & 0.91 \\
\hline \multirow{5}{*}{ Vehicle } & Mass & $1900[\mathrm{~kg}]$ \\
\cline { 2 - 3 } & Frontal Area & $0.22\left[\mathrm{~m}^{2}\right]$ \\
\cline { 2 - 3 } & Rolling Resistance Factor & 0.015 \\
\cline { 2 - 3 } & Aero Drag Factor & 0.31 \\
\cline { 2 - 3 } & Correction Coefficient of & 1.05 \\
& Rotating Mass & $0.305[\mathrm{~m}]$ \\
\cline { 2 - 3 } & Wheel Radius & \\
\hline \hline
\end{tabular}

\section{A. Assessment on IVP Method}

Figs. 6 to 11 illustrate the general comparison on velocity prediction by different methods. In the prediction, the receding forecasting manner is adopted. The prediction is repeated every $1 \mathrm{~s}$, and the results of first prediction step is applied in next time step to achieve the optimal prediction effectiveness. Figs. 6 to 11 only display the results of first prediction step in each time step. The comparison is performed on two driving cycles that are derived from real traffic environment, and the prediction length in all methods is set to $10 \mathrm{~s}$. As can be found, the developed IVP method achieves better performance than all other benchmark methods in velocity prediction. To be specific, the predicted velocities by the IVP method are more concentrated in the reference line. More centralized distribution of the predicted velocities highlights stronger capabilities in velocity forecast. In the adopted driving cycles, the vehicle operates in the low-speed range, and more prediction errors appear in Figs. 8 and 9. The prediction error of each step shown in Figs. 8 and 9 is obtained by subtracting from the real value. Figs. 10 and 11 further compare the performance of four methods in velocity prediction. The comparison with real values is illustrated by the cloud of points, which is positioned by the real speed as X-axle coordinate and predicted speed as Y-axle coordinate on the cloud map. The point locating closer to the diagonal line means better prediction efficiency. As can be seen from Figs. 10 and 11, the proposed IVP method achieves smallest prediction errors in most of time, with more points locating on or more closely to the diagonal line than other methods. In contrast, MF and iOVM behave worse than the IVP and HVPP method with more points deviating from the diagonal line. The comparison in distribution of error points on cloud maps 
better reveal the different performance in velocity prediction by four methods, justifying the application superiority of the proposed IVP method.
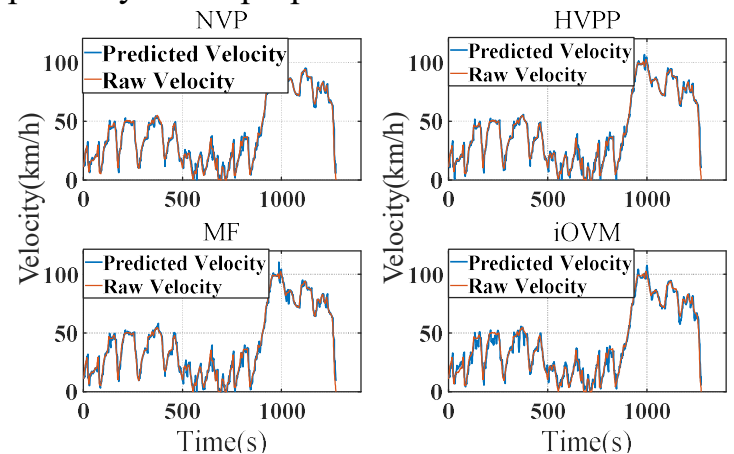

Fig. 6. General compare results of velocity prediction on driving cycle 1 .
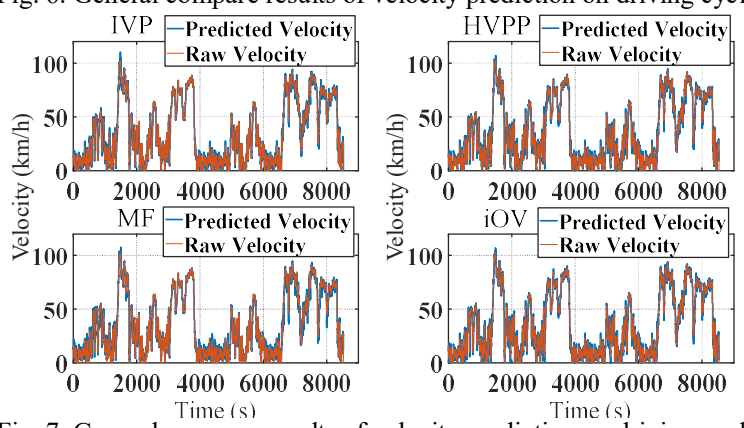

Fig. 7. General compare results of velocity prediction on driving cycle 1 .
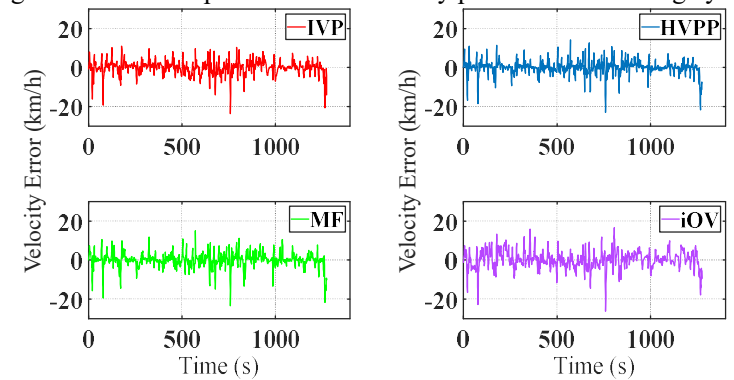

Fig. 8. Prediction error of each step on driving cycle 1.
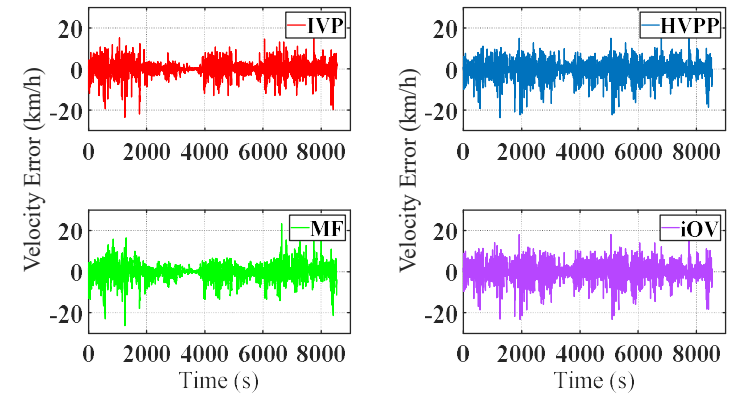

Fig. 9. Prediction error of each step on driving cycle 2.
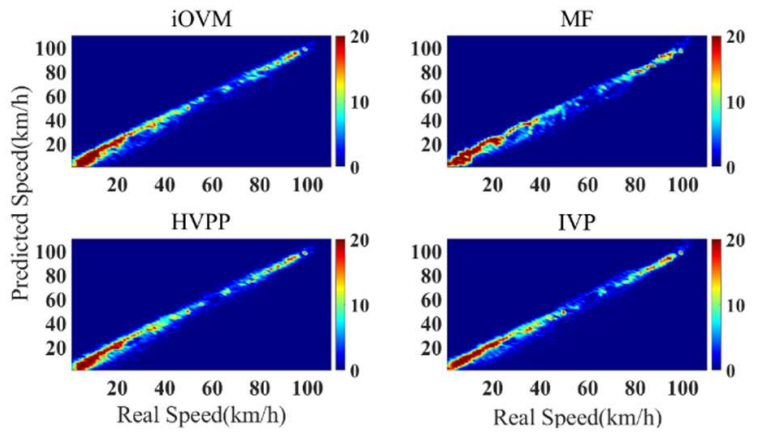

Fig. 10. Normalization prediction error of each step on driving cycle 1.
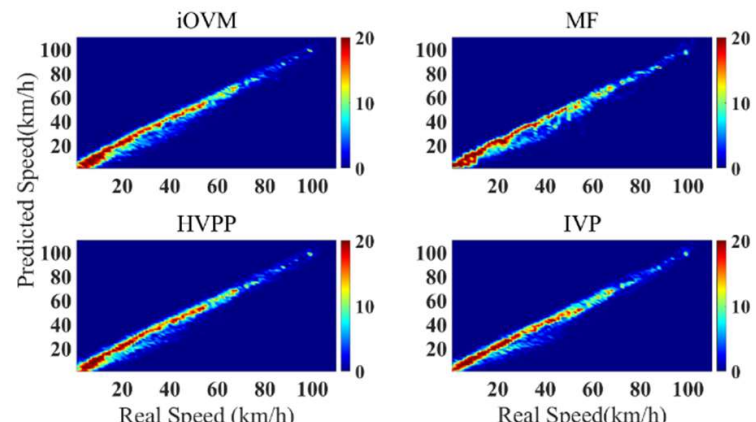

Fig. 11. Normalization prediction error of each step on driving cycle 2.

Due to the specific design, the single iOVM and single MF capture more information about the anticipated macroscopic and microscopic behaviors respectively, but lose the detail of the interactions between the macroscopic and microscopic levels. Obviously, focusing on the macroscopic behavior will only provide a cursory guide on the future velocity profiles, and excessive emphasis on the microscopic performance can also be equally misleading. Even though the HVPP method performs well, the combination of the velocity profiles through the linear regression technique results in information loss and discounts the prediction accuracy. Instead of combining different velocity profiles by a weighed function, even with the carefully tuned parameters, the proposed IVP method synthesizes the velocity profiles in frequency domain, and thus reduce information loss. Table II lists the numerical comparison among different methods. In Table II, RMSE means root means square error (RMSE), and MAE denotes mean absolute error (MAE), as:

$$
\begin{aligned}
R M S E & =\sqrt{\frac{1}{m} \sum_{i=1}^{m}\left(y_{i}-\hat{y}_{i}\right)^{2}} \\
M A E & =\frac{1}{m} \sum_{i=1}^{m}\left|\left(y_{i}-\hat{y}_{i}\right)\right|
\end{aligned}
$$

where $y_{i}$ is the estimation value, and $\hat{y}_{i}$ is the real value from the test data. The numerical results in Table II support the conclusion on the visual comparison in Figs. 6 to 11. According to the results in Table II, the raised IVP method outperforms HVPP with further enhanced accuracy in velocity prediction. The RMSE of the IVP method in the two studies is reduced by $7.17 \%$ and $9.46 \%$, compared to that by the HVPP method. Likewise, the MAE of IVP method in the two studies is reduced by $7.86 \%$ and $9.22 \%$, respectively. Unsurprisingly, the single iOVM and single MF methods incur significantly worse performance with much larger RMSE and MAE. The MAE by single $\mathrm{iOVM}$ and single MF is closer to RMSE, indicating that the average prediction error in each step is larger. Table II also lists the calculation time by different methods. The listed CPU time denotes the online computation time (without including the training time), which is a key index related to the capability of real-time implementation. From Table II, the CPU time by IVP is slightly longer than that by single iOVM and single MF. This is because the combined velocity prediction process can be completed only after the separate velocity prediction and frequency combination are executed. However, the CPU time of IVP is only around $0.2 \mathrm{~s}$, satisfying the demand for real-time 
implementation (the control step is usually $1 \mathrm{~s}$ ), even with the consideration of the communication delay in macroscopic velocity prediction and data transfer.

TABLE II

NUMERICAL COMPARISON AMONG DIFFERENT METHODS

\begin{tabular}{|c|c|c|c|c|}
\hline Group & Method & RMSE & MAE & CPU Time (s) \\
\hline \multirow{4}{*}{1} & Single iOVM & 3.613 & 2.347 & 0.131 \\
\hline & Single MF & 2.537 & 2.079 & 0.187 \\
\hline & HVPP & 1.827 & 1.501 & 0.195 \\
\hline & IVP & 1.696 & 1.383 & 0.202 \\
\hline \multirow{4}{*}{2} & Single iOVM & 3.749 & 2.199 & 0.129 \\
\hline & Single MF & 2.632 & 1.781 & 0.189 \\
\hline & HVPP & 1.998 & 1.312 & 0.198 \\
\hline & IVP & 1.809 & 1.191 & 0.206 \\
\hline \multicolumn{5}{|c|}{$\begin{array}{l}\text { TABLE III } \\
\text { SON AMONG I }\end{array}$} \\
\hline Group & Method & RMSE & MAE & CPU Time (s) \\
\hline \multirow{4}{*}{1} & BP-NN & 3.061 & 2.413 & 0.192 \\
\hline & SVM & 2.412 & 1.957 & 0.189 \\
\hline & LSTM & 1.813 & 1.632 & 0.194 \\
\hline & IVP & 1.696 & 1.383 & 0.202 \\
\hline \multirow{4}{*}{2} & BP-NN & 3.166 & 2.179 & 0.193 \\
\hline & SVM & 2.464 & 1.725 & 0.190 \\
\hline & LSTM & 1.917 & 1.306 & 0.196 \\
\hline & IVP & 1.809 & 1.191 & 0.206 \\
\hline
\end{tabular}

To prove the viability of the IVP method further convincingly, additional comparison study is addressed. The IVP method is compared with some popular methods that are preferred in the literature. Table III lists the compare results between the IVP method and the popular benchmark algorithms As can be found, the IVP method outperforms BP-NN, SVM and LSTM algorithms. The superior performance of the IVP method owes to the full-scale consideration of macroscopic and microscopic feature in future driving. The other three algorithms, trained by the same velocity, tend to reveal future driving dynamics from the macroscopic perspective, thus losing the future driving details and behaving poorer than the IVP method. The CPU time by the IVP method is quite close to other methods, showing rational online processing time.

For real-time implementation, the velocity prediction should be examined in all driving condition. Fig. 12 presents the box plot in terms of the prediction error by different methods with various forecasting lengths. The IVP method, benefiting from reasonable traffic flow analysis and superior characterized speed prediction, expresses more stable prediction accuracy with the increase of prediction horizon. The accuracy by the HVPP method in long prediction horizon is discounted due to the sensitive parameters in the linear regression function. The single iOVM and single MF behaves poorly due to improper involvement of future driving features. Tables IV and V list the test results by the HVPP and IVP methods across 60 driving cycles that are extracted from real traffic. The derived driving cycles represent different road conditions including city urban road (CR), highway (HW) and the combined road (CW) that covers both $\mathrm{CR}$ and $\mathrm{HW}$. For each driving condition, there are 20 driving cycles. In Tables IV and V, RMSE $E_{a v a}, M A E_{a v a}$ and $T_{\text {cal ava }}$ denote the average RMSE, MAE and the online computing time, respectively; $R M S E_{\text {best }}, M A E_{\text {best }}$ and
$T_{\text {cal min }}$ express the minimum RMSE, minimum MAE and minimum online computation time. The results in Tables IV and $\mathrm{V}$ demonstrate that the IVP method showcases better stability and robustness in different driving conditions. The average RMSE, MAE and online computation time by the IVP are all less than that by the HVPP, especially in HW and CW where the traffic state distribution is not even. The enhanced performance of the IVP can be attributed to the efficient combination of the macroscopic and microscopic velocity in the frequency domain. For the HVPP method, its stability and robustness in velocity prediction is weaker than that of the IVP. This mainly arises from the MC based microscopic velocity prediction and the linear regression-based combination.
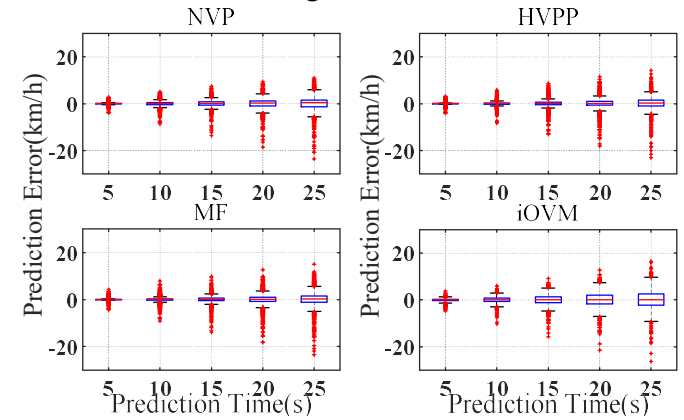

Fig. 12. Box plot in prediction errors of different methods with various forecasting horizons.

TABLE IV

STABILITY AND ROBUSTNESS ANALYSIS ON HVPP METHOD

\begin{tabular}{lllllll}
\hline \hline Type & $R M S E_{\text {ava }}$ & $M A E_{\text {ava }}$ & $T_{\text {cal ava }}$ & $R M S E_{\text {best }}$ & $M A E_{\text {best }}$ & $T_{\text {cal min }}$ \\
\hline CR & 3.931 & 2.531 & 0.196 & 3.813 & 2.343 & 0.194 \\
\hline HW & 3.977 & 2.543 & 0.199 & 3.832 & 2.491 & 0.196 \\
\hline Com & 3.966 & 2.539 & 0.198 & 3.826 & 2.502 & 0.197 \\
\hline \hline \multicolumn{7}{c}{ TABLE V } \\
\hline \hline Type & STABILITY AND ROBUSTNESS ANALYSIS ON IVP METHOD \\
\hline CR & MAE $E_{\text {ava }}$ & $T_{\text {cal_ava }}$ & $R M S E_{\text {best }}$ & $M A E_{\text {best }}$ & $T_{\text {cal_min }}$ \\
\hline HW & 3.712 & 2.236 & 0.205 & 3.684 & 2.153 & 0.201 \\
\hline Com & 3.793 & 2.322 & 0.209 & 3.707 & 2.259 & 0.202 \\
\hline \hline
\end{tabular}

B. Evaluation on Case Study results

A further evaluation of the IVP method and its ability to achieve fuel consumption savings in PHEV is addressed. The simulation based case study is performed on a driving cycle extracted from real traffic data, as shown in Fig. 13. Table VI lists the detailed comparison in energy consumption obtained by different methods. In Table VI, FC means the fuel consumption, EC denotes the electricity consumption, and EFC is the equivalent fuel consumption that includes the converted fuel consumption from the electrical energy. As can be found, it can be concluded that the IVP method further reduces the difference between the MPC based strategy and the optimum benchmark solved by the global DP. With more accurate velocity prediction, the EFC by MPC based method with IVP is reduced by $5.82 \%$, compared with that by the MPC based method with HVPP, and achieves the superior fuel economy, reaching $97.1 \%$ of the global DP optimum solution. Compared with MPC based method with HVPP, the MPC based method IVP has achieved progressive improved in PHEV energy management. The HVPP and IVP method both belong to the combining methods that integrate macroscopic and microscopic velocity into the final velocity profile of future driving. The 
HVPP has proved its ability in our previous work [16], which performs better than benchmarks that only deals with single macroscopic and microscopic velocity prediction. Compared with the HVPP method, the IVP method solves information loss in the velocity synthesis process, achieving more accurate prediction results than the HVPP method. Consequently, MPC based method with IVP surpasses MPC based method in HVPP in PHEV energy management. Despite difference in EFC saving between MPC with IVP and HVPP methods is only $5.82 \%$, it is still a remarkable improvement compared with benchmarks in the literature. The EFC by the CD-CS strategy plays worst, and increases by $26.7 \%, 34.5 \%$ and $38.6 \%$, compared with those by the MPC with HVPP, the MPC with IVP and DP. The general comparison in energy consumption verifies the positive roles of IVP in improving the effect of MPC based strategy.

To further investigate the role of IVP in improving the energy management in PHEV, some results related to vehicle energy consumption and component operation are assessed. As the MPC based method with HVPP achieves close EFC to the MPC based method with IVP, the following evaluation will only concentrate on comparing the differences between the CD-CS strategy, MPC based method with IVP and DP. The results illustrated in Fig. 13 demonstrate that the MPC based energy management strategy achieves quite similar performance to the global DP, and much better than the rule based strategy with the form of less fuel consumption and more efficient electricity utilization. In Fig. 13, the fuel and electricity consumption managed by the MPC and DP based strategy show a gradual consumption trend, revealing that the MPC based strategy cooperatively employs different power sources to propel the vehicle without firstly exhausting the electrical energy. The CD-CS strategy, however, is significantly dependent on the battery state before the vehicle falls into charging sustaining mode after $3200 \mathrm{~s}$. In the charging sustaining mode, the engine becomes the primary power source propelling the vehicle even in low speed (ultimately increasing the overall fuel consumption due to low efficiency). Consequently, the early switch into the charging sustaining mode leads to inferior fuel economy when implementing the rule-based strategy. DP, as a global optimization-based strategy, has full foresight of the route at the beginning of trip and can plan the energy distribution in an optimal manner (but obviously unsuitable for real-time implementation). Compared with the DP based method, the MPC based method with IVP can forecast the future velocity profiles by incorporating velocity prediction, which provides more beneficial reference to instruct the solution search with considering the impact on total performance in each step, thereby narrowing the gap between instantaneous optimization and global optimization. With more accurate velocity predictions incorporated into the MPC based strategy, the fuel economy of PHEV can be improved further.

TABLE VI

ENERGY CONSUMPTION BY DIFFERENT METHODS

\begin{tabular}{llll}
\hline \hline Method & FC (g) & EC $(\mathbf{k W h})$ & EFC (L/100km) \\
\hline Original CS & 1923.1 & 3.62 & 4.13 \\
\hline MPC with HVPP & 851.2 & 3.09 & 3.26 \\
\hline MPC with IVP & 841.1 & 3.14 & 3.07 \\
\hline DP & 749.1 & 3.17 & 2.98 \\
\hline \hline
\end{tabular}

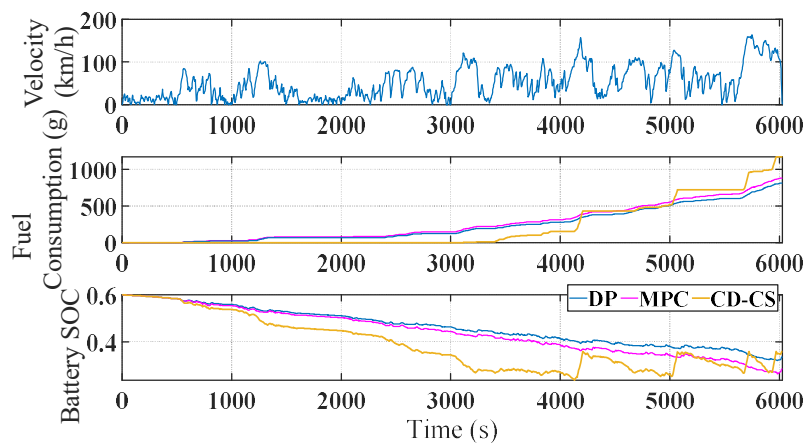

Fig. 13. The fuel consumption and SOC trajectories by different methods.

To further understand how the IVP is enhancing the control effect of the MPC based method, some extra results showing the status of powertrain operation are plotted in Figs. 14 to 17. Figs. 14 and 15 illustrate the performance of ICE, motor and generator by different strategies. In line with the conclusions on Fig. 13, the MPC with IVP method governs the powertrain components to behave closely to that instructed by DP. The operation frequencies and quantity of ICE, motor and generator are similar, compared with the MPC based method with IVP and DP. The main differences among various strategies are particularly apparent after $3200 \mathrm{~s}$. The vehicle following the CD-CS strategy falls into the charging sustaining stage at this point. The ICE has to provide most of the required tractive energy. As shown in Fig. 15, ICE, motor and generator operates dramatically different with those by MPC with IVP method and DP during $5000 \mathrm{~s}$ to $5100 \mathrm{~s}, 5700 \mathrm{~s}$ to $5800 \mathrm{~s}$, and $5900 \mathrm{~s}$ to 6000 s. Together with the operation modes shown in Figs. 16 and 17 , the reason leading to the difference can be explained below. At $5000 \mathrm{~s}$ to $5100 \mathrm{~s}$, the MPC with IVP and DP order the vehicle to operate in serial and parallel assist mode in CD stage, while the CD-CS enables the vehicle to operate in serial and parallel charge mode in the CS stage limited by the slight available electricity. At $5700 \mathrm{~s}$ to $5800 \mathrm{~s}$, the MPC with IVP and DP control vehicle operate in EV mode, serial assist mode and parallel assist mode in CD stage, while the CD-CS orders the frequent operation switch between the $\mathrm{CD}$ and $\mathrm{CS}$ stage. At $5900 \mathrm{~s}$ to $6000 \mathrm{~s}$, the vehicle by the MPC with IVP and DP still operates in CD stage, while the vehicle by the CD-CS scheme encounters the mode switch between CD and CS a few times. The accurate sensing future driving dynamics by the IVP method provides MPC algorithm rational and accurate reference in solution searching. With the precisely predicted velocity profile, MPC algorithm can optimally distribute energy within powertrains, avoiding electricity prematurely consumed by enabling components operate in high efficiency zones.

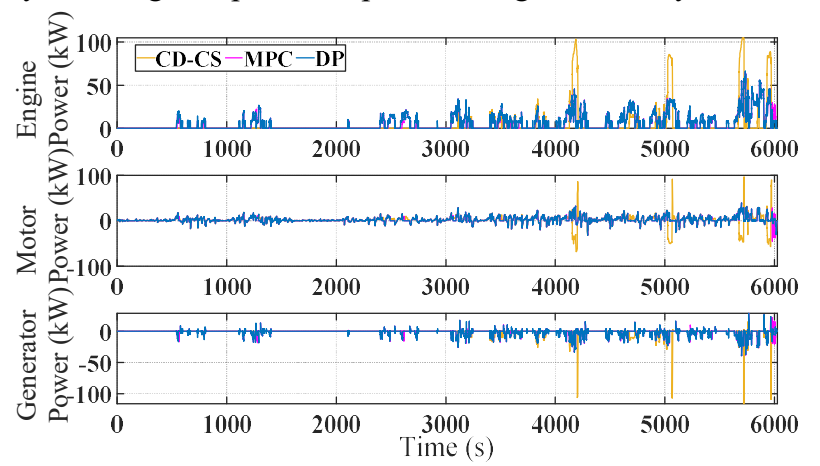

Fig. 14. The ICE, motor and generator performance by different methods. 


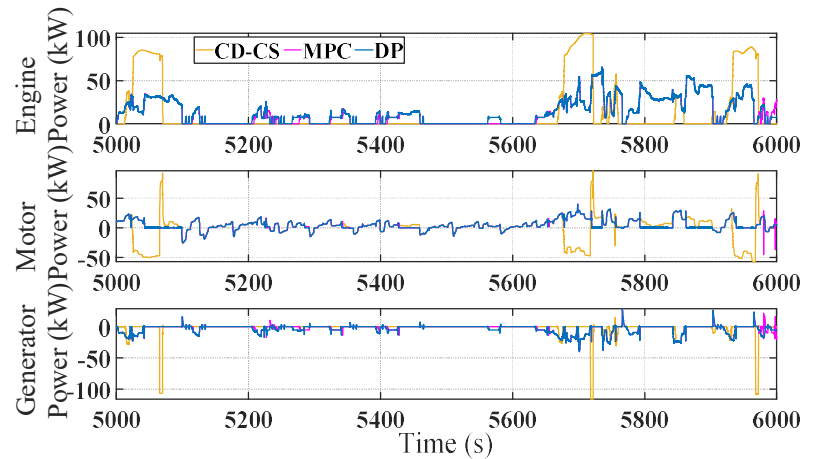

Fig. 15. The zoomed-in ICE, motor and generator performance by different methods.

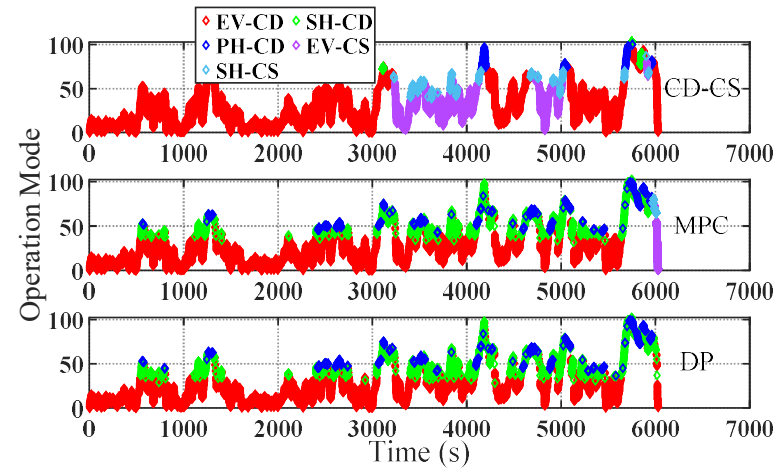

Fig. 16. The illustration on operation modes by different methods.

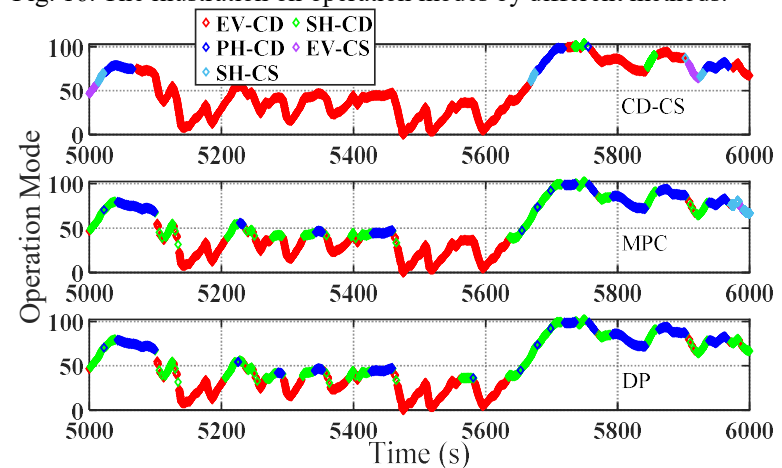

Fig. 17. The zoomed-in operation modes by different methods.

Figs. 18 to 20 show the operating efficiencies of the different power components by different strategies. The 2D comparison in operation efficiency reveals the inner mechanism why EFC by MPC based method with IVP and DP is better than the simple rule based strategy. The MPC based method with IVP and DP regulate the powertrain components generally to operate in regions with better efficiency, contributing to better fuel economy. The efficient operation of the components is realized by reasonably distributing the energy among power sources after accurately forecasting future driving conditions, avoiding frequent mode switch and ICE on/off. The validation on the raised IVP method through a series of comparison study shows that remarkable improvement in prediction accuracy can be achieved compared with the HVPP method. The evaluation on the case study demonstrates the critical roles of IVP in promoting fuel economy of PHEV. In short, the IVP method represents one of the promising applications raised by IoVs.

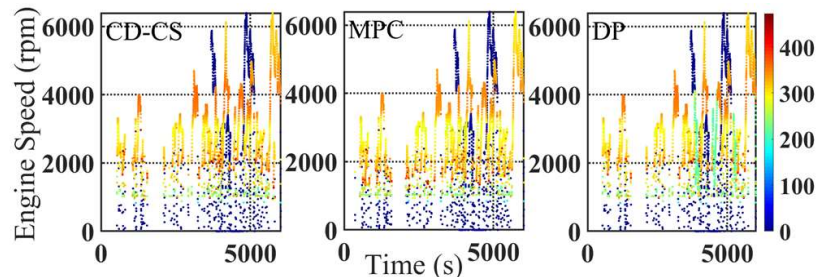

Fig. 18. The illustration on 2D ICE efficiencies by different methods.

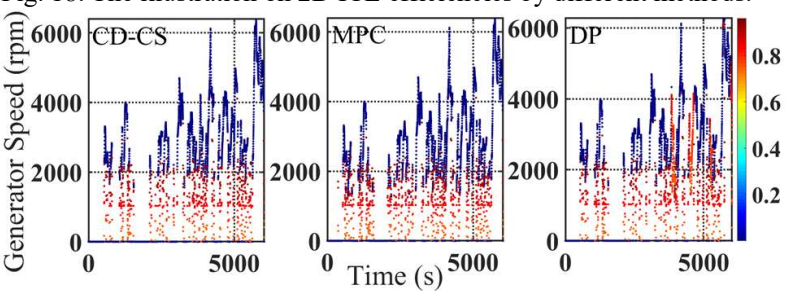

Fig. 19. The illustration on 2D generator efficiencies by different methods.
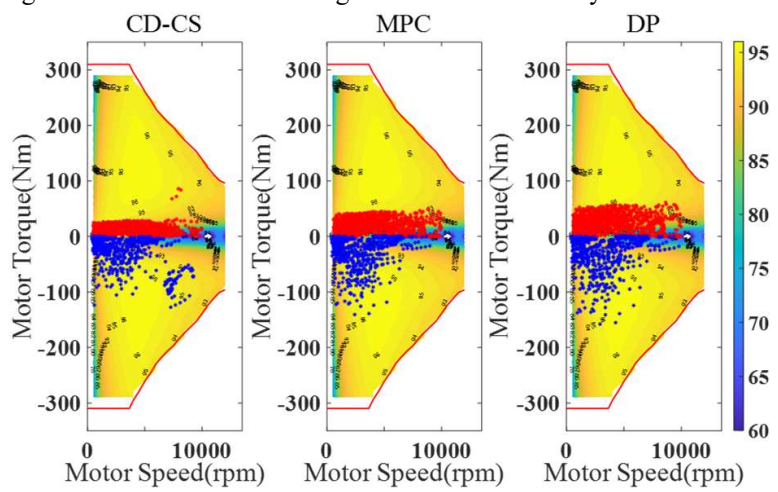

Fig. 20. The illustration on motor operation points by different methods.

\section{Assessment through Hardware-in-Loop Test}

To validate the performance of the raised method and its functionality in vehicle-environment co-operation based case application, a hardware-in-loop (HIL) test is conducted, which, as illustrated in Fig. 20, underpins the test by cooperatively incorporating three host PCs and one real-time controller. The MPC based control strategy is compiled in host PC 1 and downloaded to the real-time dSpace based controller. The IVP based velocity profile forecast is completed in host PC 1 , and the vehicle model, included component sub-models, is executed in host PC 2. The test scenario is supplied by host PC 1 . The macroscopic and microscopic velocity prediction is respectively completed in host $\mathrm{PC} 1$ and $\mathrm{PC} 3$. The communication between the controller and host PCs is attained via CAN bus communication.

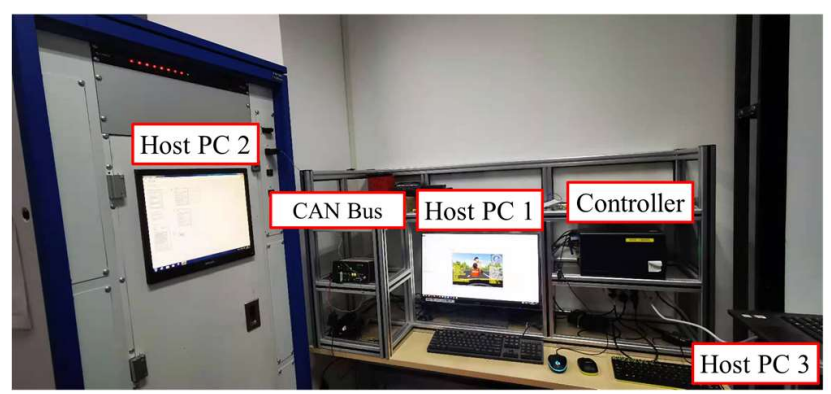

Fig. 21. HIL test platform.

TABLE VII

NUMERICAL RESULTS COMPARISON IN HIL TEST

\begin{tabular}{llllll}
\hline \hline Type & $R M S E_{\text {ava }}$ & MAE ava & $T_{\text {total_ava }}$ & $T_{\text {total_macro }}$ & $T_{\text {total_micr }}$ \\
\hline
\end{tabular}




\begin{tabular}{llllll}
\hline CR & 3.715 & 2.233 & 0.204 & 0.032 & 0.141 \\
\hline HW & 3.789 & 2.323 & 0.210 & 0.031 & 0.142 \\
\hline Com & 3.762 & 2.312 & 0.207 & 0.031 & 0.141 \\
\hline
\end{tabular}

Table VII lists the numerical results obtained from the HIL test, wherein Ttot_a, Ttot_mac_a and Ttot_mic_a denote the total step time cost, time cost in macroscopic velocity prediction and time cost in miscrscopic velocity prediction, respectively. Except providing the total average computation time of IVP based velocity prediction in each step, Table VII shows the separate average time cost in macroscopic and microscopic velocity prediction in each step. As can be detected, the macroscopic and microscopic velocity prediction by the IVP based method is all less than the total step cost in various conditions, demonstrating that the designed method can support real-time application. As the velocity combination in frequency domain also costs a fringe of time, there exist a little difference between the total step time cost and the sum of time cost in macroscopic and microscopic velocity prediction. Additionally, the proposed IVP method, in line with simulation results, keeps promising stability in prediction accuracy in real-time environment. It is noteworthy that the promising performance is attained on the assumption that no disturbance on macroscopic velocity prediction emerges from multi-rate situation of GNSS. The location by global navigation satellite system (GNSS) may lead to large pseudorange errors under multi-rate measurement, which may affect statistics of vehicles on certain route segment and discredit velocity prediction precision. To mitigate the adverse effect from GNSS, the improved Kalman filter [39] and a map matching algorithm [40] has been exploited to tackle these issues of the multi-rate GNSS and pseudorange measurements.

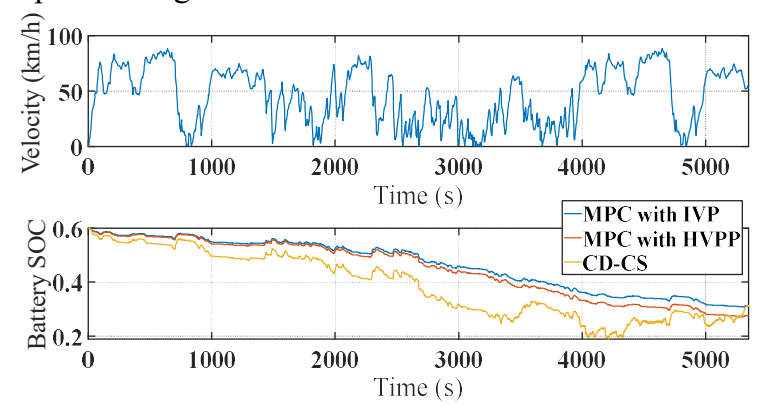

Fig. 22. The velocity profiles and SOC trajectories by different methods in HIL test.

TABLE VIII

ENERGY CONSUMPTION BY DIFFERENT METHODS

\begin{tabular}{llll}
\hline \hline Method & FC (g) & EC (kWh) & EFC (L/100km) \\
\hline Original CS & 1749.7 & 3.27 & 4.11 \\
\hline MPC with HVPP & 766.1 & 2.78 & 3.23 \\
\hline MPC with IVP & 761.2 & 2.82 & 3.05 \\
\hline
\end{tabular}

Fig. 22 and Table VIII present the results of MPC based application after incorporating the IVP method. A new driving cycle, as shown in Fig. 22, is imported in the HIL test, where the MPC based method with IVP achieves the optimal performance in energy saving among three methods. Compared with the CD-CS scheme, the MPC based method with HVPP performs more closely to the MPC based method with IVP, and the results are generally in line with results in simulation. In HIL test, the MPC based method IVP respectively attains 5.6\% and $25.8 \%$ improvement in energy saving, compared with the MPC based method with HVPP and CD-CS method. The better potential in energy saving benefits from the endowed future driving information predicted by the IVP method. Thus, one can conclude that clear understanding on future driving conditions can facilitate the MPC based algorithm to optimally search rational solutions that contribute to better fuel economy by controlling the powertrain operation in high-efficiency area.

\section{Discussion}

The developed IVP method is evaluated in both simulation and HIL test, and the results showcase the improved performance, compared with those of a series of benchmark algorithms including the HVPP method proposed in our former work [16]. The investigation on compare studies indicates that the cooperative consideration of macro and micro impact on future driving can contribute to more precise velocity prediction than the ones that focus individually on macroscopic or microscopic future driving behaviors. The combination of the predicted macroscopic and microscopic velocity in frequency domain mitigates the valuable information loss that might happen in linear regression-based methods in our former work [16], thus refining the forecasting accuracy further. The role of the raised method in vehicle-environment co-operation is examined through a case study, and the results manifest that the proposed method can enhance the implementation of vehicleenvironment co-operation into PHEV energy management method by enabling high-performance predictive control.

The novel IVP method only considers vehicle longitude driving behaviors in a stable driving vehicle swarm. The vehicles in the swarm are randomly distributed on route segment, rather than forming a platoon with car-following mode by hard constraints. To extend application scenarios in the future, lateral velocity prediction will be integrated into the IVP method, for better applications related to automatic driving. In the velocity prediction considers vehicle lateral dynamics, two main concerns need to be carefully tackled. Firstly, the estimation of vehicle slip angle in lane changing needs to be considered. The vehicle slip angle is critical in lateral velocity estimation, of which the process may be affect by errors include GPS irrational errors. To ascertain velocity prediction accuracy, advanced sideslip estimators [41-42] should be incorporated into the extended IVP method. Secondly, the accuracy of GNSS based location may be influenced by the loss of reception and possible large biases/attack. The adverse impact might be more obvious in autonomous platoon scenario [43]. Therefore, the enhanced IVP should efficiently account for faculty location and external intervene with the help of the adaptive learning and control methods [43-44]. This is a research direction in future work.

\section{CONCLUSION}

In this paper, a novel velocity prediction method is proposed by taking advantage over the advanced IoVs. The proposed method comprehensively considers the impact on future driving from macroscopic and microcosmic perspectives. With the assist from mobile edge computing framework under IoVs, future macroscopic and microcosmic velocity is respectively forecasted by the improved optimal velocity model and Mondrian forest in different time domain. The acquired macroscopic and microcosmic velocities are combined into 
final predicted velocity in frequency domain to avoid important information loss. Compared with the state-of-the-art research, the RMSE and MAE of the presented novel method in velocity prediction can be reduced by $3.42 \%$ and $6.82 \%$. To further validate performance of the novel velocity prediction method and clearly identify its key role in vehicle-environment cooperation, a case study about energy management strategy of PHEV is performed. The novel velocity prediction method is incorporated into the MPC based strategy. The case study results show that the effect of MPC based strategy is promoted after incorporating the novel velocity prediction method, thus highlighting its superior performance and pivotal roles in vehicle-environment cooperation from another point of view.

In the future work, more efforts will be continuously devoted to macroscopic and microcosmic velocity prediction. The macroscopic velocity prediction will be focused on designing advanced methods to fuse more traffic information. Meanwhile, the methods to improve long-time microscopic velocity prediction will also be carefully investigated.

\section{REFERENCES}

[1] Z. Tian, X. Gao, S. Su, J. Qiu, X. Du, and M. Guizani, "Evaluating Reputation Management Schemes of Internet of Vehicles Based on Evolutionary Game Theory," IEEE Transactions on Vehicular Technology, Article vol. 68, no. 6, pp. 5971-5980, 2019, Art no. 8685209, doi: 10.1109/TVT.2019.2910217.

[2] Y. Dai, D. Xu, S. Maharjan, G. Qiao, and Y. Zhang, "Artificial Intelligence Empowered Edge Computing and Caching for Internet of Vehicles," IEEE Wireless Communications, Article vol. 26, no. 3, pp. 1218, 2019, Art no. 8752477, doi: 10.1109/MWC.2019.1800411.

[3] B. Cao, L. Zhang, Y. Li, D. Feng, and W. Cao, "Intelligent Offloading in Multi-Access Edge Computing: A State-of-the-Art Review and Framework," IEEE Communications Magazine, Review vol. 57, no. 3 , pp. 56-62, 2019, Art no. 8663993, doi: 10.1109/MCOM.2019.1800608.

[4] H. Peng, Q. Ye, and X. Shen, "Spectrum Management for Multi-Access Edge Computing in Autonomous Vehicular Networks," IEEE Transactions on Intelligent Transportation Systems, Article vol. 21, no. 7, pp. 3001-3012, 2020, Art no. 8748212, doi: 10.1109/TITS.2019.2922656

[5] J. Guo, H. He, and C. Sun, "ARIMA-based road gradient and vehicle velocity prediction for hybrid electric vehicle energy management," IEEE Transactions on Vehicular Technology, Article vol. 68, no. 6, pp. 5309-5320, 2019, Art no. 8695855, doi: 10.1109/TVT.2019.2912893.

[6] Y. Zhou, A. Ravey, and M. C. Péra, "A survey on driving prediction techniques for predictive energy management of plug-in hybrid electric vehicles," Journal of Power Sources, Review vol. 412, pp. 480-495, 2019, doi: 10.1016/j.jpowsour.2018.11.085.

[7] Y. Zhang et al., "Energy management strategy for plug-in hybrid electric vehicle integrated with vehicle-environment cooperation control," Energy, Article vol. 197, 2020, Art no. 117192, doi 10.1016/j.energy.2020.117192

[8] C. Sun, S. J. Moura, X. Hu, J. K. Hedrick, and F. Sun, "Dynamic Traffic Feedback Data Enabled Energy Management in Plug-in Hybrid Electric Vehicles," IEEE Transactions on Control Systems Technology, Article vol. 23, no. 3, pp. 1075-1086, 2015, Art no. 6930758, doi: 10.1109/TCST.2014.2361294.

[9] C. Yang, S. You, W. Wang, L. Li, and C. Xiang, "A Stochastic Predictive Energy Management Strategy for Plug-in Hybrid Electric Vehicles Based on Fast Rolling Optimization," IEEE Transactions on Industrial Electronics, Article vol. 67, no. 11, pp. 9659-9670, 2020, Art no. 8917923, doi: 10.1109/TIE.2019.2955398.

[10] C. Sun, X. Hu, S. J. Moura, and F. Sun, "Velocity Predictors for Predictive Energy Management in Hybrid Electric Vehicles," IEEE Transactions on Control Systems Technology, Article vol. 23, no. 3, pp. 1197-1204, 2015, Art no. 6917015, doi: 10.1109/TCST.2014.2359176.

[11] F. Zhang, X. Hu, R. Langari, and D. Cao, "Energy management strategies of connected HEVs and PHEVs: Recent progress and outlook," Progress in Energy and Combustion Science, Review vol. 73, pp. 235-256, 2019, doi: 10.1016/j.pecs.2019.04.002.

[12] E. Hyeon, Y. Kim, N. Prakash, and A. G. Stefanopoulou, "Influence of speed forecasting on the performance of ecological adaptive cruise control," in ASME 2019 Dynamic Systems and Control Conference, DSCC 2019, 2019, vol. 1, doi: 10.1115/DSCC2019-9046.

[13] Y. Xing and C. Lv, "Dynamic State Estimation for the Advanced Brake System of Electric Vehicles by Using Deep Recurrent Neural Networks," IEEE Transactions on Industrial Electronics, Article vol. 67, no. 11, pp. 9536-9547, 2020, Art no. 8906229, doi: 10.1109/TIE.2019.2952807.

[14] L. Li, S. Coskun, F. Zhang, R. Langari, and J. Xi, "Energy Management of Hybrid Electric Vehicle Using Vehicle Lateral Dynamic in Velocity Prediction," IEEE Transactions on Vehicular Technology, Article vol. 68, no. 4, pp. 3279-3293, 2019, Art no. 8629961, doi: 10.1109/TVT.2019.2896260.

[15] Y. Chen, C. Hu, and J. Wang, "Motion Planning with Velocity Prediction and Composite Nonlinear Feedback Tracking Control for Lane-Change Strategy of Autonomous Vehicles," IEEE Transactions on Intelligent Vehicles, Article vol. 5, no. 1, pp. 63-74, 2020, Art no. 8910360, doi: 10.1109/TIV.2019.2955366

[16] Y. Zhang, L. Chu, Y. Ou, C. Guo, Y. Liu, and X. Tang, "A CyberPhysical System-Based Velocity-Profile Prediction Method and Case Study of Application in Plug-In Hybrid Electric Vehicle," IEEE Transactions on Cybernetics, pp. 1-12, 2019, doi: 10.1109/TCYB.2019.2928945.

[17] J. Wang, J. Hu, G. Min, W. Zhan, Q. Ni, and N. Georgalas, "Computation Offloading in Multi-Access Edge Computing Using a Deep Sequential Model Based on Reinforcement Learning," IEEE Communications Magazine, Article vol. 57, no. 5, pp. 64-69, 2019, Art no. 8713801, doi: 10.1109/MCOM.2019.1800971.

[18] A. Ndikumana, N. H. Tran, D. H. Kim, K. T. Kim, and C. S. Hong, "Deep Learning Based Caching for Self-Driving Cars in Multi-Access Edge Computing," IEEE Transactions on Intelligent Transportation Systems, pp. 1-16, 2020, doi: 10.1109/TITS.2020.2976572.

[19] X. Gao, L. Dai, and A. M. Sayeed, "Low RF-Complexity Technologies to Enable Millimeter-Wave MIMO with Large Antenna Array for 5G Wireless Communications," IEEE Communications Magazine, Article vol. 56, no. 4, pp. 211-217, 2018, doi: 10.1109/MCOM.2018.1600727.

[20] M. Zeng, M. Zeng, W. Hao, O. A. Dobre, and H. V. Poor, "Delay minimization for massive MIMO assisted mobile edge computing," IEEE Transactions on Vehicular Technology, Article vol. 69, no. 6, pp. 6788-6792, 2020, Art no. 9027954, doi: 10.1109/TVT.2020.2979434.

[21] Hasebe, Katsuya, Akihiro Nakayama, and Yūki Sugiyama. "Dynamical model of a cooperative driving system for freeway traffic." Physical review E 68.2 (2003): 026102

[22] Z. Ning, J. Huang, X. Wang, J. J. P. C. Rodrigues, and L. Guo, "Mobile Edge Computing-Enabled Internet of Vehicles: Toward Energy-Efficient Scheduling," IEEE Network, Article vol. 33, no. 5, pp. 198-205, 2019 , Art no. 8786080, doi: 10.1109/MNET.2019.1800309.

[23] Bishop, Gary, and Greg Welch. "An introduction to the kalman filter." Proc of SIGGRAPH, Course 8.27599-23175 (2001): 41

[24] Y. Qi, L. Tian, Y. Zhou, and J. Yuan, "Mobile Edge Computing-Assisted Admission Control in Vehicular Networks: The Convergence of Communication and Computation," IEEE Vehicular Technology Magazine, Article vol. 14, no. 1, pp. 37-44, 2019, Art no. 8584057, doi: 10.1109/MVT.2018.2883336.

[25] W. Laghari, M. Baloch, M. Mengal, and S. Shah, "Performance Analysis of Analog Butterworth Low Pass Filter as Compared to Chebyshev TypeI Filter, Chebyshev Type-II Filter and Elliptical Filter," Circuits and Systems, vol. 05, pp. 209-216, 01/01 2014, doi: 10.4236/cs.2014.59023.

[26] A. Liaw and M. J. R. N. Wiener, "Classification and Regression by randomForest," vol. 23, no. 23, 2002.

[27] Lakshminarayanan, Balaji, Daniel M. Roy, and Yee Whye Teh. "Mondrian forests for large-scale regression when uncertainty matters." Artificial Intelligence and Statistics. PMLR, 2016.

[28] Mourtada, Jaouad, Stéphane Gaïfas, and Erwan Scornet. "Minimax optimal rates for Mondrian trees and forests." Annals of Statistics 48.4 (2020): 2253-2276.

[29] Mourtada, Jaouad, Stéphane Gaïffas, and Erwan Scornet. "Universal consistency and minimax rates for online mondrian forests." arXiv preprint arXiv:1711.02887 (2017).

[30] K. S. Thyagarajan, Introduction to digital signal processing using MATLAB with application to digital communications (Introduction to Digital Signal Processing using MATLAB with Application to Digital Communications). 2018, pp. 1-505. 
[31] D. Takahashi, Fast Fourier Transform Algorithms for Parallel Computers. 2019.

[32] Saleh, Alfa, Khairani Puspita, and Andi Sanjaya. "Implementation of Equal Width Interval Discretization on SMARTER Method for Selecting Computer Laboratory Assistant." 2018 6th International Conference on Cyber and IT Service Management (CITSM). IEEE, 2018.

[33] Yuanjian, Zhang, et al. "An Optimal Control Strategy Design for Plugin Hybrid Electric Vehicles Based on Internet of Vehicles." Energy (2021): 120631.

[34] P. E. Gill, W. Murray, and M. A. Saunders, "SNOPT: An SQP algorithm for large-scale constrained optimization," SIAM Review, Review vol. 47, no. 1, pp. 99-131, 2005, doi: 10.1137/S0036144504446096.

[35] Lin, Xinyou, Zhaorui Wang, and Jiayun Wu. "Energy management strategy based on velocity prediction using back propagation neural network for a plug - in fuel cell electric vehicle." International Journal of Energy Research 45.2 (2021): 2629-2643.

[36] Liu, Jin-peng, et al. "Forecasting of wind velocity: An improved SVM algorithm combined with simulated annealing." Journal of Central South University 20.2 (2013): 451-456.

[37] Altché, Florent, and Arnaud de La Fortelle. "An LSTM network for highway trajectory prediction." 2017 IEEE 20th International Conference on Intelligent Transportation Systems (ITSC). IEEE, 2017.

[38] Hongwen, He, et al. "Real-time global driving cycle construction and the application to economy driving pro system in plug-in hybrid electric vehicles." Energy 152 (2018): 95-107.

[39] Yuan, Hao, et al. "A novel model-based internal state observer of a fuel cell system for electric vehicles using improved Kalman filter approach." Applied Energy 268 (2020): 115009.

[40] Zhang, Dongqing, Yucheng Dong, and Zhaoxia Guo. "A turning pointbased offline map matching algorithm for urban road networks." Information Sciences 565 (2021): 32-45

[41] Hashemi, Ehsan, et al. "Fault tolerant consensus for vehicle state estimation: A cyber-physical approach." IEEE Transactions on Industrial Informatics 15.9 (2019): 5129-5138

[42] Selmanaj, Donald, et al. "Vehicle sideslip estimation: A kinematic based approach." Control Engineering Practice 67 (2017): 1-12.

[43] He, Xingkang, Ehsan Hashemi, and Karl H. Johansson. "Secure platooning of autonomous vehicles under attacked GPS data." arXiv preprint arXiv:2003.12975 (2020).

[44] Jin, Xu, et al. "An adaptive learning and control architecture for mitigating sensor and actuator attacks in connected autonomous vehicle platoons." International Journal of Adaptive Control and Signal Processing 33.12 (2019): 1788-1802.

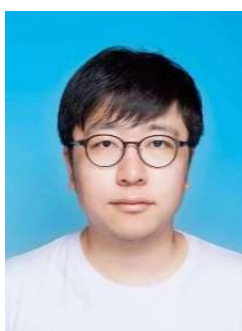

Yuanjian Zhang received the M.S. degree in Automotive Engineering from the Coventry University, UK, in 2013 and the Ph.D. degree in Automotive Engineering from Jilin University, China, in 2018. Then he joined the University of Surrey, Guildford, UK, as a Research Fellow in advanced vehicle control. In early 2019 , he secured a Research Fellow position in Sir William Wright Technology Centre, Queen's University Belfast, UK.

$\mathrm{He}$ is currently a Lecturer (Assistant Professor) with the Department of Aeronautical and Automotive Engineering, Loughborough University, Loughborough, UK. He has authored several books and over 50 peer-reviewed journal papers and conference proceedings. His current research interests include advanced control on electric vehicle powertrains, vehicle-environment-driver cooperative control, vehicle dynamic control, and intelligent control for driving assist system.

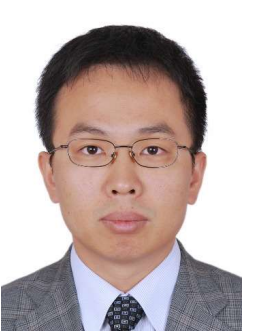

Zheng Chen received the B.S. and M.S. degrees in electrical engineering and the Ph.D. degree in control science engineering from Northwestern Polytechnical University, Xi'an, China, in 2004, 2007, and 2012, respectively. He was a Post-Doctoral Fellow and a Research Scholar with the University of Michigan, Dearborn, MI, USA, from 2008 to 2014.

$\mathrm{He}$ is currently a Professor with the Faculty of Transportation Engineering, Kunming University of Science and Technology, Kunming, Yunnan, China and also a Marie-Curie Research Fellow with the School of Engineering and Materials Science, Queen Mary University of London, London, UK. He has conducted over 30 projects and has published over 100 peer-reviewed journal papers and conference proceedings. His research interests include battery management system, battery status estimation, and energy management of hybrid electric vehicles. He is a Fellow of the Institution of Engineering and Technology. In addition, he is a recipient of the Yunnan Oversea High Talent Project, China, and the second place of IEEE VTS Motor Vehicles Challenge in 2017 and 2018.

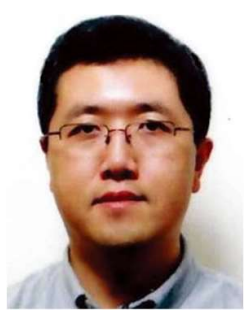

Guang $\mathbf{L i}$ received the Ph.D. degree in electrical and electronics engineering, specialized in control systems, from the University of Manchester, Manchester, U.K., in 2007.

He is currently a Reader in Dynamics Modeling and Control with the Queen Mary University of London, London, U.K. His research interests include constrained optimal control, model predictive control, adaptive robust control and control applications including renewable energies, energy storage, etc.

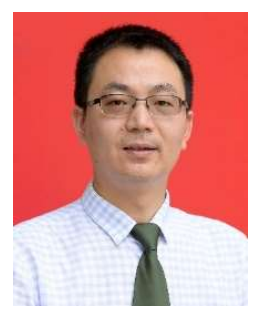

Yonggang Liu, IEEE Senior Member, was born in Chongqing, China in 1982. He received the B.S and $\mathrm{PhD}$ degrees in Automotive engineering from Chongqing University, Chongqing, China, in 2004 and 2010, while he was a joint $\mathrm{PhD}$ of University of Michigan-Dearborn, MI, USA, from 2007 to 2009.

Now he is a Professor and Doctoral supervisor, Dean Assistant with School of Automotive Engineering, Chongqing University. He is also a Committeeman of Technical Committee on Vehicle Control and Intelligence of Chinese Association of Automation (CAA). His research interests mainly include optimization and control of intelligent Electric Vehicles (EV/HEV) power system, and integrated control of vehicle Automatic Transmissions. He has leaded more than 20 research projects, such as National Natural Science Foundation of China (both Youth Fund and General Program), Ph.D. Programs Foundation of Ministry of Education of China, and China Postdoctoral Science Foundation. More than 70 research papers have been published and 10 patents have been awarded. He served as the head of the secretariat in The International Conference on Power Transmissions (ICPT2016) and the session chairman of the International Symposium on Electric Vehicles (ISEV2017), etc.

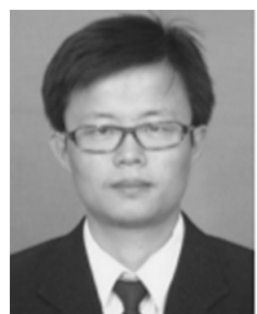

Yanjun Huang received the Ph.D. degree in mechanical and mechatronics engineering from the University of Waterloo, Waterloo, Canada, in 2016.

He is a Professor with the School of Automotive Studies, Tongji University, Shanghai, 201804, China. He has authored several books and over 50 papers in journals and conferences. His research interests include the vehicle holistic control in terms of safety, energy saving, and intelligence, including vehicle dynamics and control, hybrid electric vehicle/electric vehicle optimization and control, motion planning and control of connected and autonomous vehicles, and human-machine cooperative driving. Dr. Huang serves as the Associate Editor and Editorial Board Member for the IET Intelligent Transport System, Society of Automotive Engineers (SAE) International Journal of Commercial vehicles, International Journal of Vehicle Information and Communications, Automotive Innovation, etc.

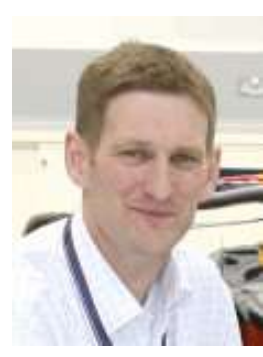

Geoff Cunningham received the Bachelor's degree and $\mathrm{PhD}$ degree in mechanical engineering from the Queen's University of Belfast. He is a Member of the Institution of Mechanical Engineers and a Chartered Engineer.

He is currently with the School of Mechanical and Aerospace Engineering, Queen's University Belfast. Dr. Cunningham has been involved in automotive research for 28 years. His initial work was a combination of fundamental research on internal combustion engines and applied design at the subsystem and vehicular level. But he has moved over recent years to the design of hybrid and electric vehicles. Now he focuses on the development of electric powertrains for passenger buses in the Sir William Wright Technology Centre, where he is supervising three $\mathrm{PhD}$ students. 


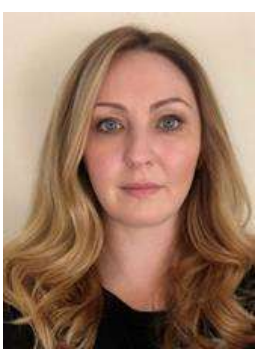

Juliana Early received the $\mathrm{PhD}$ degree from the University of Glasgow in 2006 and joined the academic staff in the School of Mechanical and Aerospace Engineering at Queen's University Belfast in 2007.

Now, she is a Professor and Chartered Engineer at Queen's University Belfast. Her current interests lie in clean propulsion technologies, including design of hybrid, electric and hydrogen powertrain and optimisation strategies. She has published over 80 research publications and has led a range of research programmes funded through EU FP6, FP7, Horizon 2020, Innovate UK and UKRI EPSRC. 